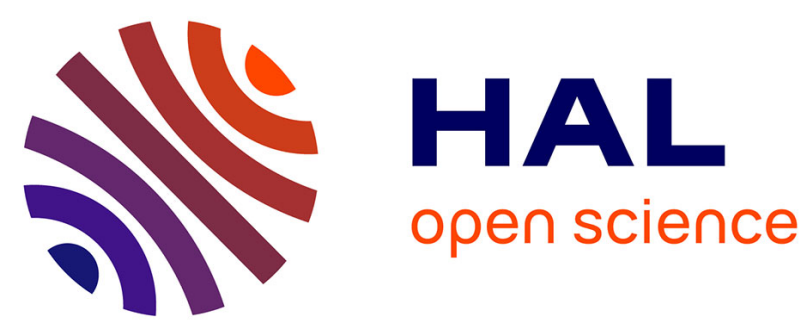

\title{
Mutualistic mimicry enhances species diversification through spatial segregation and extension of the ecological niche space
}

Thomas G. Aubier, Marianne Elias, Violaine Llaurens, Nicolas Chazot

\section{- To cite this version:}

Thomas G. Aubier, Marianne Elias, Violaine Llaurens, Nicolas Chazot. Mutualistic mimicry enhances species diversification through spatial segregation and extension of the ecological niche space. Evolution - International Journal of Organic Evolution, 2017, 71 (4), pp.826 - 844. 10.1111/evo.13182 . hal-01525954

\section{HAL Id: hal-01525954 \\ https://hal.sorbonne-universite.fr/hal-01525954}

Submitted on 22 May 2017

HAL is a multi-disciplinary open access archive for the deposit and dissemination of scientific research documents, whether they are published or not. The documents may come from teaching and research institutions in France or abroad, or from public or private research centers.
L'archive ouverte pluridisciplinaire HAL, est destinée au dépôt et à la diffusion de documents scientifiques de niveau recherche, publiés ou non, émanant des établissements d'enseignement et de recherche français ou étrangers, des laboratoires publics ou privés. 


\title{
Mutualistic mimicry enhances species diversification through spatial segregation and extension of the ecological niche space
}

\author{
Thomas G. Aubier ${ }^{1,2 *}$, Marianne Elias², Violaine Llaurens ${ }^{2}$ and Nicolas Chazot ${ }^{2,3}$
}

1. Centre d'Ecologie Fonctionnelle et Evolutive, CEFE - UMR 5175 - CNRS, Université de Montpellier, EPHE, Université Paul Valéry, 1919 route de Mende, F-34293, Montpellier 5, France

2. Institut de Systématique, Evolution, Biodiversité, ISYEB - UMR 7205 - CNRS, MNHN, UPMC, EPHE, Muséum national d'Histoire naturelle, Sorbonne Universités, 57 rue Cuvier, CP50, F-75005, Paris, France

3. Department of Biology, Lund University, Lund, Sweden

* Corresponding author

\section{Corresponding author :}

Thomas G. Aubier:

thomas.aubier@normalesup.org

+33637313230

Centre d'Ecologie Fonctionnelle et Evolutive, 1919 route de Mende, 34090, Montpellier, France

Keywords: Phylogenetic trees, range size, macroevolutionary patterns, mutualism, Müllerian mimicry, agentbased model.

Running head: Mutualistic mimicry and macroevolutionary pattern

Number of tables: 3

Number of figures: 5

Number of words: 7500

Manuscript format: LaTeX 


\section{Abstract}

Species richness varies among clades, yet the drivers of diversification creating this variation remain poorly understood. While abiotic factors likely drive some of the variation in species richness, ecological interactions may also contribute. Here, we examine one class of potential contributors to species richness variation that is particularly poorly understood: mutualistic interactions. We aim to elucidate large-scale patterns of diversification mediated by mutualistic interactions using a spatially-explicit population-based model. We focus on mutualistic Müllerian mimicry between conspicuous toxic prey species, where convergence in colour patterns emerges from predators' learning process. To investigate the effects of Müllerian mimicry on species diversification, we assume that some speciation events stem from shifts in ecological niches, and can also be associated with shift in mimetic colour pattern. Through the emergence of spatial mosaics of mimetic colour patterns, Müllerian mimicry constrains the geographical distribution of species and allows different species with similar ecological niches to exist simultaneously in different regions. Müllerian mimicry and the resulting spatial segregation of mimetic colour patterns thus generate more balanced phylogenetic trees and increase overall species diversity. Our study sheds light on complex effects of Müllerian mimicry on ecological, spatial and phylogenetic diversification. 


\section{INTRODUCTION}

One of the most remarkable macroevolutionary patterns in biology is the heterogeneity of species richness observed across the tree of life. Factors that generate and shape such variation between clades are numerous and are still debated among evolutionary biologists. Species diversity within a clade results from the balance between speciation and extinction rates (Rabosky 2013), which are known to be affected by biogeographical processes, such as geological (Newell 1952; Peters 2005) and climatic features (Erwin 2009; Figueirido et al. 2012; Fraser et al. 2015). In addition to abiotic factors, ecological interactions can also shape macroevolutionary patterns of diversity (Erwin 2000; Jablonski 2007, 2008a; Cavender-Bares et al. 2009) through their effects on speciation (Schluter 1994; Dieckmann and Doebeli 1999; Bolnick 2004; Grant and Grant 2006) and extinction rates (Weiner 1990; Law et al. 1997). For instance, biotic interactions like competition (Darwin 1871; Rabosky 2013), hostpathogens coevolutionary dynamics (Ricklefs 2010) or predation (Sepkoski 1978) have been suggested to explain the dampening of diversity trajectories observed in many phylogenies at the macroevolutionary scale (Rabosky and Lovette 2008; McPeek 2008; Phillimore and Price 2008).

While the impact of competitive and other antagonistic interactions on diversification has been extensively explored (Ehrlich and Raven 1964; Rabosky 2013), the effect of mutualistic interactions - i.e. beneficial interactions between species - on diversification is still debated (Jablonski 2008a; Hembry et al. 2014; Althoff et al. 2014). On the one hand, mutualism may help to create or maintain isolation between populations interacting with phenotypically different partners (Kiester et al. 1984). Changes in coevolved traits could lead to reproductive isolation of the locally coevolving populations, resulting in matching speciation events. Such co-diversification has been shown mostly empirically on mutualistic symbioses (Gómez and Verdú 2012; Litsios et al. 2012; Joy 2013) and plant-pollinators interactions (Hodges 1997; Sargent 2004) - yet, phylogenies frequently display significant mismatches between interacting species (see Althoff et al. 2014, for instance). On the other hand, while antagonistic interactions drive evolutionary arms races, mutualisms lead to stabilizing selection because of positive frequency-dependent selection. This stabilizing selection might reduce phenotypic diversity within populations, thus hindering the process of diversification via ecological speciation (Kopp and Gavrilets 2006; Yoder and Nuismer 2010). Overall, the effect of mutualistic interactions on species diversification remains unclear.

Here we investigate the impact of a mutualistic interaction, Müllerian mimicry, on the process of diversification. Müllerian mimicry is a local selective process that drives convergent evolution of warning signals among unpalatable species, because defended species that exhibit a locally abundant warning signal benefit from reduced predation risk (Müller 1879; Mallet and Joron 1999). This mutualistic relationship is observed in a wide variety of taxonomic groups and involves interactions both within and among clades. Phylogenies of mimetic clades have indeed revealed convergent evolution of warning colour patterns between both closely and 
distantly-related species in a large panel of taxa, such as butterflies (Brower 1996; Jiggins et al. 2006), frogs (Symula et al. 2001), birds (Dumbacher and Fleischer 2001), bumblebees (Williams 2007), catfishes (Alexandrou et al. 2011) and velvets ants (Wilson et al. 2012). Mutualistic mimicry may affect the diversification process. Indeed, Müllerian mimicry has been hypothesized to favour speciation through mating preference associated with mimetic colour pattern (Jiggins et al. 2001; Merrill et al. 2011) and strong selection pressure against non-mimetic hybrids (Mallet and Barton 1989; Jiggins 2008; Merrill et al. 2012). Shifts in warning colour patterns therefore promote reproductive isolation between subpopulations via both pre- and post-zygotic barriers (Chamberlain et al. 2009). Müllerian mimicry also favours speciation through ecological adaptation as shifts in mimetic colour patterns have been shown to be associated with shifts in host plant (Willmott and Mallet 2004), microhabitat (Elias et al. 2008) and even altitudinal niche (Chazot et al. 2014). However, derived species whose colour patterns do not match warning signals displayed by locally abundant defended species suffer from high attack rate by predators (Mallet and Barton 1989; Kapan 2001; Chouteau et al. 2016). Therefore, although shift in colour pattern can promote speciation, the establishment and the persistence of derived species displaying different warning colour patterns are limited by predation. While the role of shift in mimetic colour patterns in the initiation of speciation is well documented, the resulting effect of Müllerian mimicry on the diversification processes at macroevolutionary scale warrants investigation.

By promoting local convergence in warning patterns, Müllerian mimicry greatly affects the spatial distribution of species displaying different colour patterns. Geographical divergence of mimetic patterns resulting in a spatial mosaic of warning signals is observed in most mimetic species including moths (Sbordoni et al. 1979), butterflies (Sheppard et al. 1985; Brower 1996), birds (Dumbacher and Fleischer 2001), frogs (Symula et al. 2001), bumblebees (Williams 2007), millipedes (Marek and Bond 2009) and velvet ants (Wilson et al. 2012). Spatial mosaics of mimetic patterns result from the combination of the opposite effects of (1) positive frequencydependent selection favouring convergent mimetic patterns at local scale and (2) stochastic processes such as genetic drift allowing new colour pattern to establish (shifting balance hypothesis) (Mallet 2010; Chouteau and Angers 2011). Theoretical approaches describing the formation of a spatial mosaic of mimetic forms usually assume relaxed predation pressure on rare warning signals through absence of predators (Sherratt 2006) or predators' neophobic responses (Aubier and Sherratt 2015). Once the spatial mosaic of warning signal is established, colour shifts can be promoted if they represent adaptations to habitats/microhabitats with different mimetic communities (Papageorgis 1975; Chouteau et al. 2016). Shift in colour pattern can also be favoured when the abundance of non-toxic mimics becomes too large: defended prey may benefit from increased protection when shifting toward better defended mimetic communities (Franks et al. 2008; Aubier et al. in press). Overall, selection acting on warning signals constrains the spatial distribution of mimetic species and has an important influence on species range. 
Species range properties may exert positive or negative influences on diversification (Jablonski and Roy 2003). Limited range size is a powerful predictor of extinction probability (Kiessling and Aberhan 2007; Jablonski 2008b). However the effect of range size on speciation rate is less intuitive. On the one hand, species with large range size should be less likely to speciate by vicariance - i.e. to split into isolated subpopulations because of geographical barriers - because the existence of a contact zone is more likely if the species range size is large in the first place (Pigot et al. 2010; Moen and Morlon 2014). On the other hand, larger ranges should increase the opportunity to encounter new habitats, which could promote reproductive isolation between populations through local adaptation. In addition, large population sizes should harbour higher standing genetic variation on which selection can act, thereby favouring the emergence of new adaptive traits that may promote ecological speciation (Birand et al. 2012). Ecological interactions are known to favour the generation and the maintenance of parapatric distributions (Bull 1991), which limit dispersal and geographic range expansion over evolutionary time (Pigot and Tobias 2013). Such ecological effects on species range size could greatly affect per-lineage rates of speciation and extinction, and therefore macroevolutionary patterns, in a similar way as competitive exclusion (Ranjard et al. 2014; Moen and Morlon 2014). This calls for spatially-explicit models to investigate the effect of mutualistic interactions such as Müllerian mimicry on species diversification.

Here we use a theoretical approach to jointly assess the influence of Müllerian mimicry on (1) the geographical distribution of mimetic species and (2) the diversification process. We extend a population genetic model used to investigate the emergence of mosaics of mimetic morphs (Sherratt 2006) to a macroevolutionary scale by implementing speciation events. We consider either ecological speciation events where niche divergence can lead to sympatric speciation, or allopatric speciation without niche divergence. A proportion of these events are also set to be associated with shifts in colour pattern. Therefore, we do not investigate the already welldescribed effect of Müllerian mimicry on the evolution of reproductive barriers, but we investigate the effect of colour shift on spatial, temporal and phylogenetic diversification. Since many speciation events result in immediate extinction because of negative selection by predators or competitive exclusion, we record only effective speciation events, whereby species persist for at least a fixed number of generations. We track extinction and effective speciation events to generate phylogenies, and we analyze the resulting spatial and phylogenetic macroevolutionary patterns. We specifically study the influence on diversification patterns of (i) the proportion of speciation events associated with shift in mimetic pattern and (ii) the number of possible mimetic morphs to tackle the following questions: (1) Does Müllerian mimicry constrain species range sizes? (2) Does Müllerian mimicry promote species diversification at the macroevolutionary scale? (3) What is the influence of the spatial distribution of mimetic morphs and species on phylogenetic macroevolutionary patterns? 


\section{THE MODEL}

\section{Purpose}

We investigate how Müllerian mimicry affects geographical and phylogenetic diversification of a clade. To link local processes and macroevolutionary patterns in an explicit spatial context, we develop an agent-based model. We simulate the invasion of a spatial grid by toxic species exhibiting colour patterns used as warning signals by predators. Individuals of a species can locally diverge and form a new species. Two models of speciation are considered: (1) sympatric ecological speciation and (2) allopatric speciation. When speciation is sympatric, the population living within a cell is split in two, one half still belonging to the ancestral species and the other half belonging to a new species occupying a different ecological niche. When speciation is allopatric, the entire population living within a cell becomes isolated and forms a new species, without ecological niche divergence. In both cases, speciation can also be accompanied by a shift in colour pattern with a certain probability. Since many speciation events result in immediate extinction, we track only effective speciation events, i.e. cases where the derived species persists for at least a chosen number of generations.

We perform simulations (1) with predation, whereby natural selection favours colour pattern convergence (mimicry) and (2) without predation, which corresponds to a neutral model without selection promoting mimicry. Neutral expectations regarding mimicry are also simulated assuming a null proportion of speciation to be associated with shift in colour pattern.

The model is implemented in $\mathrm{C}++$ and the code is available upon request. Table 1 sums up parameters notations and provides the default values implemented in the simulations.

\section{State variables and scale}

The model comprises two hierarchical levels: grid cells and species. We build a spatially-explicit grid-based model in which space is represented by a regular $G \times G$ lattice of cells. Each cell can contain populations of conspicuous and toxic organisms belonging to different species.

Species are distributed across the spatial grid. They are characterized by the state variables: morph (i.e. colour pattern), and position in the ecological niche space. The morph $m_{i}$ of species $i$ is a discrete number with $m_{i} \in\left\{1,2, \ldots N_{\text {morph }}\right\}$ assuming $N_{\text {morph }}$ as the number of possible morphs. Morphs are used as warning cues by predators during associative learning. The ecological niche occupied by species $i$ is described by its position $z_{i}$ in a two-dimensional space that represents quantitative characters determining resource use. The niche occupied by a species is thus a point with coordinates $z_{i}=\left(x_{i}, y_{i}\right)$ in the niche space with $\left(x_{i}, y_{i}\right) \in \mathbb{R}^{2}$ and $\left(x_{i}, y_{i}\right) \in[0,1]^{2}$. The carrying capacity of each species in any given cell is equal to $K$. However, the density reached by each species in any cell is determined by local interspecific competition for resources (see below). 
We use discrete time steps, corresponding to non-overlapping generations.

\section{Process overview and scheduling}

Within each time step, several phases are processed in the following order: local catastrophes (extinction of all populations in a locality), migration, predation, speciation (which may cause effective speciation), reproduction, competition for resources. After reproduction, parents are completely replaced by their offspring. These environmental and population processes are described in the subsection "Submodels" below.

\section{Design concepts}

Interactions. - Within cell, populations of different species are competing for resources. The intensity of competition between two species depends on the proximity of their occupied ecological niches. In addition to this antagonistic interaction, predation and predators' learning process - characterized by the avoidance of prey with a colour pattern known to be associated with toxicity - lead to mutualism between coexisting species sharing the same morph. Mimetic species occurring in the same cell therefore suffer from a reduced per capita attack rate, since they share the cost associated with predators' learning.

Stochasticity. - All environmental and population processes are probabilistic. Some probabilities are defined as parameters of the model (probabilities of local catastrophes, migration, presence of predators, speciation, shift in colour pattern). Predation and survival rate are computed from the composition of the prey community.

Emergence. - The maintenance of a population in a cell depends on the local community of species. For instance, a local population belonging to a species is more likely to persist in a cell if it shares a mimetic morph already settled in the cell (thereby sharing the cost of learning of predators), or if it exploits a different ecological niche (thereby lowering competition). Species range then emerges from the ability of the species to persist and expand in multiple cells.

In each cell, when predators are present, a dominant morph becomes rapidly established. Individuals carrying a rare morph then suffer from a high per capita attack risk. A patch is defined as contiguous cells where individuals share the same morph regardless of the species they belong to. Spatial mosaics of morphs - composed of multiple patches - can emerge, due to stochastic processes allowing new colour patterns to settle.

At the evolutionary scale, effective speciation rate emerges from the state variables of the diverging subpopulation (i.e. occupied ecological niche and morph) and from the species composition of the cell where divergence happens. 
Observation. - We record the cell occupancy by each species, the state variable of each species (occupied ecological niche and morph) and the resulting phylogeny at the end of each simulation.

\section{Initialization}

At initial state, we introduce a single species in a corner of the grid since an invasion process following local introduction has been shown to be the only scenario leading to the emergence of morph diversity with the model of predation implemented here (presented below) (Sherratt 2006). Yet, the exact position of the initial species in the grid does not change our results (Fig. S1, available online).

\section{Submodels}

Local catastrophes - Local catastrophes cause extinction of all populations occupying a cell with a probability $p_{d}$ at any given time step. These stochastic events purge species that have not invaded multiple cells.

Migration. - Each individual can migrate with a probability $\mu$ to one of the eight neighbouring cells (King's move). Borders of the lattice are assumed to be reflective so edge cells have fewer neighbouring cells.

Predation. - Predators are not explicitly modelled, and we only implement predation events on the community of prey. We assume that a community of predators is present with probability $p_{p}$ in each cell of the grid. Stochasticity of predator presence in each cell allows new colour patterns to invade through relaxed predation, as hypothesized in Mallet's (2010) shifting balance theory. At each time step, the community of predators

- when present - samples $\tau_{\max }$ individuals per morph, which enables predators to associate given morphs to toxicity and to subsequently avoid prey carrying these morphs (Mallet and Joron 1999; Sherratt 2006). Each morph is treated independently by the community of predators, and individuals carrying an abundant morph therefore benefit from a low attack risk.

Speciation and effective speciation. - Speciation of any species can occur locally with probability $p_{s}$ per cell. Speciation happens either in sympatry (with probability $s_{\text {symp }}$ ) or in allopatry (with probability $1-s_{\text {symp }}$ ). When sympatric speciation occurs, half of the individuals from species $i$ in the cell become reproductively isolated from the other half and form a new species $j$. The derived species $j$ occupies a different ecological niche $z_{j}=\left(x_{j}, y_{j}\right)$ derived from its ancestral one. The new ecological traits $\left(x_{j}, y_{j}\right)$ are drawn from a normal distribution with means equal to the ancestral ecological traits $\left(x_{i}, y_{i}\right)$ and with standard deviation $\sigma_{N}$. When allopatric speciation occurs, all individuals from species $i$ in the cell form a new species $j$, and occupy the same position in the ecological niche space $\left(z_{j}=z_{i}\right)$. 
To study the effect of shift in colour pattern on the diversification process, a speciation event can be accompanied by a colour pattern shift as follows. When a speciation event occurs with probability $p_{s}$ (either in sympatry or in allopatry), a species $i$ leads to the formation of a new species $j$ whose individuals harbour either (1) the same colour pattern (referred hereafter as speciation without colour shift, $m_{j}=m_{i}$ ) with probability $s_{n}$ or $(2)$ a different colour pattern (referred as speciation with colour shift) with probability $s_{c}\left(s_{n}+s_{c}=1\right)$. When a colour shift occurs, the morph $m_{j}$ of the new species is drawn from a uniform distribution in the discrete interval $\left\{1, \ldots, N_{\text {morph }}\right\}$ until $m_{j} \neq m_{i}$. The total probability of speciation per species per cell $p_{s}$ is set to be constant, whatever the proportion $s_{\text {symp }}$ of ecological speciation in sympatry and the proportion $s_{c}$ of speciation associated with colour shift. Our aim is not to investigate the impact of colour shift on the process of speciation, but rather to test the influence of the emergence of new warning patterns on diversification.

Depending on their characteristics (colour pattern, ecological niche, initial density), newly diverged species may frequently go extinct after a small number of generations because of competition, predation or catastrophic events. To avoid any confounding effect of such high species turnover, we thus consider only species persisting for more than 500 generations in the summary statistics (changing this threshold does not affect qualitatively our results, data not shown). When a speciation event produces a species that persists for more than 500 generations, we consider such event as effective speciation (similarly to models of protracted speciation, Rosindell 2010).

Reproduction. - Reproduction is asexual and we assume equal fertility for every individual, producing $b$ offspring per time step. Parents are completely replaced by their offspring, so that generations do not overlap.

Competition for resources. - Offpring survival depends on the carrying capacity of the cell and on the competition among individuals, which itself depends on their ecological niche. It is calculated using an analogue of the Beverton-Holt model: population growth is logistic as a consequence of density-dependent resource competition. Offspring belonging to species $i$ thus survive to the reproduction stage with probability $v_{i}$ :

$$
v_{i}=\frac{1}{1+(b-1) \frac{\sum_{j \in\{1, \ldots S\}} \alpha_{i j} N_{j}}{K}}
$$

where $b$ is the number of offspring per individual, $S$ the number of species in the cell, $\alpha_{i j}$ the competition term between species $i$ and species $j$ calculated from their ecological niche distance and $N_{j}$ the number of individuals from species $j . K$ corresponds to the carrying capacity of each species. A species can reach its carrying capacity $K$ when there is no competing species. Otherwise, it reaches a lower density due to interspecific competition (see next paragraph). $K$ is the same for all species, and its value has been chosen arbitrarily.

The competition term $\alpha_{i j}$ between species $i$ and $j$ is defined as follow: 


$$
\alpha_{i j}=\max \left[1-C d_{i j}{ }^{f}, \alpha_{\min }\right]
$$

where $C$ and $f$ are chosen constants, $d_{i j}=\sqrt{\left\|z_{i}-z_{j}\right\|^{2}}=\sqrt{\left(x_{i}-x_{j}\right)^{2}+\left(y_{i}-y_{j}\right)^{2}}$ is the Euclidian distance between the species' positions in the two-dimensional niche space, and $\alpha_{\min }$ is the minimum competition term possible (Fig. S2).

$\alpha_{i j}$ represents the niche overlap between two species. $\alpha_{i j}$ decreases with the niche distance $d_{i j}$ between species. Thus, there is more competition between individuals from the same species (intraspecific competition,

$\alpha_{i i}=1$ ) than between individuals from different species (interspecific competition). We choose to implement a bell-shaped relationship $(f>1)$ between $\alpha_{i j}$ and $d_{i j}$, so that competition between species occupying similar niches is very intense $\left(\alpha_{i j} \simeq 1\right)$, but then rapidly decreases when niche distances between species increases. Because we model the diversification of a clade where species are relatively similar phylogenetically, it is realistic to assume a positive competition term constrained to be greater than $\alpha_{\min }$.

\section{SIMULATION EXPERIMENTS AND STATISTICS}

\section{Parameterization}

Simulations end after five million generations, allowing sufficient time for equilibrium to be reached (Fig. S3 and S4). We implement a grid size $G=20$. Doubling the grid size does not qualitatively change results (Fig. S5 and S6).

The default value of $s_{\text {symp }}$ is 1 - i.e. speciation always occurs in sympatry with shift in ecological niche. Speciation driven by ecological adaptation in sympatry is supported by theoretical and empirical evidence in phytophagous butterflies (Ehrlich and Raven 1964; McBride and Singer 2010) and particularly in mimetic butterflies (Rosser et al. 2015). Moreover, we obtain very close macroevolutionary patterns with $s_{\mathrm{symp}}=0.5$ in supplementary analyses (Fig. S7).

The simple model of predation implemented generates positive frequency-dependent selection on prey warning signals. Although the underlying model of predator sampling behaviour has been challenged by some empirical studies (Beatty et al. 2004; Rowland et al. 2010b), positive frequency-dependent selection has been extensively documented in nature (see Chouteau et al. 2016, for instance). Here, we implement $\tau_{\max }=10$. This value is chosen because it leads to intermediate levels of frequency-dependent selection. If positive frequencydependent selection is too strong, new morphs are strongly counter-selected. If positive frequency-dependent selection is too weak, the invasion of the grid is too fast and new morphs do not have time to settle in empty 
cells. In both cases, it gives little opportunity to observe the emergence of spatial mosaics of morphs (Fig. S5).

In mimetic clades of butterflies (Jiggins et al. 2006), velvet ants (Wilson et al. 2012) and catfishes (Alexandrou et al. 2011), it is common to observe sister species that either share or diverge on colour pattern (due to stochastic or non-stochastic mechanisms listed in the Introduction), which suggests that $s_{c}$ is not skewed toward 0 or 1 in nature. We therefore implement values of $s_{c}$ ranging from 0 to 0.75 .

A few dozen of morphs are typically observed across entire mimetic clades. For instance, Williams (2007) used data for workers of all of the world's known social bumblebee species from the genus Bombus and described 24 mimicry rings. Likewise, Wilson et al. (2012) assigned 60\% of North American Dasymutilla species (velvet ants) to 6 mimicry rings. Chazot et al. (2014) documented 26 mimicry patterns on a subsample of Ithomiine species (butterflies) representing nearly $40 \%$ of the diversity of the mimetic tribe Ithomiini. The observed number of morphs does not represent the maximum number of possible morphs, but it is probably a good indicator. We therefore implement values of $N_{\text {morph }}$ ranging from 4 to 30 .

\section{Statistics}

To characterize the geographic patterns of diversification, we measure (1) species' range sizes and (2) the number of patches in the resulting spatial mosaic. (1) Species range size is defined as the number of cells occupied by each species. (2) A patch of the spatial mosaic is defined as contiguous cells where individuals share the same morph regardless the species they belong to. To increase the likelihood that the patches observed at the end of the simulation are not transient, we record only patches with a size greater than 5 cells. In our simulations, polymorphism within cell does not occur so that the patches of the mosaic are well defined.

We also record global and local species richnesses, corresponding to the total number of species existing in the grid and the mean number of species per cell, respectively. To understand the mechanisms behind the formation of spatial mosaics with different patchiness, we record the generation at which the first diversified patch (i.e. patch with two species) appears and the generation at which every cell in the grid is occupied by at least one individual.

To analyze how species occupy the ecological niche space, we measure the pairwise Euclidian distances between the species' positions in the two-dimensional niche space $\left(\forall(i, j) \in \mathbb{N}^{2}, d_{i j}\right)$. We compute the Hartigans' dip test statistic (Hartigan and Hartigan 1985; Freeman and Dale 2013) to assess the multimodality intensity in the distribution of pairwise niche distances, using the package diptest in R ( R Development Core Team 2012). When species uniformly occupy the niche space, then the dip statistic of the distribution of the pairwise niche 
distances is close to 0 . The dip statistic increases when species distribution throughout the niche space departs from uniform expectations, resulting in clustered, uneven coverage of niche space.

We build the phylogeny by retaining only branches leading to species present at the end of the simulation. We measure tree imbalance, temporal shift in diversification rate and proportion of convergence events. Imbalance refers to the distribution of branching events among sub-clades: diversification rates that differ between subclades can lead to unbalanced trees, while homogeneous rates lead to balanced trees. We measure the normalized imbalance $I_{\mathrm{C}}$ of the reconstructed trees based on the Colless' index $I_{\text {Colless }}$ (Colless 1982), which has been evaluated as one of the best estimators of tree imbalance (Kirkpatrick and Slatkin 1993; Agapow and Purvis 2002):

$$
I_{\text {Colless }}=\sum_{(\text {all interior nodes })}\left|T_{R}-T_{L}\right|
$$

Where at each node, the right and left branches subtend $T_{R}$ and $T_{L}$ tips respectively. To compare trees with different sizes, we compute the normalized Colless' index $I_{\mathrm{C}}$ under the Yule model (pure birth process) (Blum et al. 2006):

$$
I_{\mathrm{C}}=\frac{I_{\text {Colless }}-n \log (n)-n\left(\gamma_{\mathrm{E}}-1-\log 2\right)}{n}
$$

With Euler's constant $\gamma_{\mathrm{E}}=0.58$ and $n$ the number of extant species. Yule trees display $I_{\mathrm{C}}=0$, whereas trees more unbalanced than Yule trees have $I_{\mathrm{C}}>0$ and trees more balanced than Yule trees have $I_{\mathrm{C}}<0$.

We measure temporal shifts in diversification rate $(\rho)$ as the proportional difference in rate between the first $\left(r_{1}\right)$ and second half $\left(r_{2}\right)$ of the reconstructed phylogeny:

$$
\rho=\frac{r_{2}-r_{1}}{r_{1}+r_{2}}
$$

Rates are computed following Magallón and Sanderson (2001): $r=\left(\log \left(n_{e}\right)-\log \left(n_{i}\right)\right) / t$, with $n_{i}$ and $n_{e}$ the number of species at the start and the end of the time period, respectively, and $t$ the length of the period. $\rho=0$ indicates a constant diversification rate, $\rho>0$ a speedup in diversification rate and $\rho<0$ a slowdown in diversification rate. Similarly to Pigot et al. (2010), we prefer this method to the $\gamma$ statistic (Pybus and Harvey 2000), which is sensitive to species richness (McPeek 2008).

We also record the proportion of events of colour pattern convergence observed on the reconstructed phylogeny - i.e. when a speciation event with colour shift leads to phenotypic convergence between the new derived species and already existing non-sister species. 


\section{Simulations conducted}

We perform simulations without predation $\left(p_{p}=0\right.$ instead of 0.9$)$ to characterize patterns of diversification in the absence of mimetic interactions (since mimicry emerges from predators' learning process). We also perform simulations assuming a null proportion of speciation to be associated with shift in colour pattern $\left(p_{p}=0.9\right.$ and $\left.s_{c}=0\right)$.

Using multivariate linear models based on least-square estimation, we test the effect of varying levels of two factors, proportion of speciation events associated with colour pattern shift $\left(s_{c}=\{0.25,0.50,0.75\}\right)$ and number of possible morphs $\left(N_{\text {morph }}=\{4,6,8,10,20,30\}\right)$, on diversification and ecological statistics (Global species richness, Local species richness, Median of species range size, Hartigans' dip statistic, Proportion of convergence events, $I_{\mathrm{C}}, \rho$ ). To investigate the effect of spatial distribution of mimetic morphs, the number of patches of the resulting mosaics is then included as another predictor in these multivariate linear models. Effect sizes of regression coefficients are estimated using t-tests. Figures only display macroevolutionary patterns obtained with $s_{c}=0.50$.

For each combination of parameter values tested, we perform 150 simulations. 


\section{RESULTS}

Diversification patterns in the absence of predation $\left(p_{p}=0\right)$ are similar to those with predation and speciation without colour shift $\left(p_{p}=0.9\right.$ and $\left.s_{c}=0\right)$ (Fig. 3). Therefore, we can assume that variation in $s_{c}$ captures the effects of Müllerian mimicry in the model. To avoid redundancy, here we do not describe the diversification patterns in the absence of predation, since they are similar to those generated under $s_{c}=0$.

\section{Formation of the spatial mosaic of morphs}

In our model, a stable spatial mosaic of morphs frequently emerges when speciation events with colour shift are implemented (in about $50 \%$ of the simulations with $s_{c}=0.5$, see an example of simulation in Fig. 1B). Derived species with non-mimetic morphs are generally excluded from already occupied cells because they suffer from a high per capita predation risk compared to already established species that benefit from mimetic protection. Therefore, derived species whose colour pattern shifted during speciation are more likely to settle in empty cells rather than in already occupied cells. This plays a key role in the invasion of the grid and therefore in the formation of spatial mosaics of morphs. The number of patches of the resulting mosaic increases with the number of possible morphs $\left(N_{\text {morph }}\right.$ ) (inversely proportional to the probability of convergence to an existing morph) (Fig. 2B). Note that the number of patches of the resulting mosaic does not exceed the lowest value of $N_{\text {morph }}$ tested $(=4)$.

Such effect of $N_{\text {morph }}$ on the formation of the mosaic is explained by the dynamics of the invasion process. The number of patches of the resulting mosaic greatly depends on the probability of emergence of a patch with two coexisting species (Fig. 2A). When two species occupying distinct ecological niches coexist within a cell, the number of individuals in that cell is higher (up to twice the individual carrying capacity $K$ if there is no competition between those two species). From such cells, more migrants (= migration rate $\times$ the number of individuals in the cell) invade the surrounding cells leading to a quicker invasion of the grid. Derived species with exotic morphs have then less time to invade empty cells, limiting the probability of establishment of new patches with different morphs. Such acceleration in the invasion dynamic can even lead to the collapse of neighbouring patches occupied by a single species. When a speciation event is not associated with colour shift, the derived species displays the same colour pattern as its ancestral species sharing the same cell. Thus, it benefits from protection against predation because of Müllerian mimicry. Furthermore, the settlement of such co-mimetic derived species in co-occurrence with its ancestral species is favoured because the derived species can benefit from relaxed competition with its ancestral species when speciation is associated with niche divergence (i.e. simulations without allopatric speciation, $s_{\mathrm{symp}}=1$ ). When $s_{c}=0.5$, half of the speciation events are associated to colour shift. The formation of a two-species patch is therefore more likely if there is a high probability of convergence to a morph already settled in the community. Therefore, under such conditions, the 
high convergence rate generated by the low number of possible morphs $N_{\text {morph }}$ leads to a quicker grid invasion (Fig. 2A) and to a mosaic with a low number of patches (Fig. 2B). Overall, the effect of $N_{\text {morph }}$ on the patchiness of the mosaic is qualitatively the same than in Fig. $2 \mathrm{~B}$ where $s_{c}=0.5$ - yet there is no effect of $N_{\text {morph }}$ on the patchiness when $s_{c}$ is too low (e.g. $\left.s_{c}=0.25\right)$ (Fig. S8).

The formation of a mosaic is very sensitive to other parameters linked to predation (number of prey attacked before predation on a given morph ceases, $\tau_{\max }$ ) or stochastic events (such as the probability of catastrophic events, $p_{d}$ ) as already noticed in the previous two-species model (Sherratt 2006) (Fig. S5). Furthermore, the probability of speciation $\left(p_{s}\right)$, the probability of allopatric speciation without ecological shift $\left(1-s_{\text {symp }}\right)$ and the competition intensity (minimum competition term, $\alpha_{\min }$, and curvature of the competition function, $(f, C)$ ) can also influence the formation of the mosaic of morphs (Fig. S5 and S9). Decreasing the probability of speciation (low $p_{s}$ leads to fewer speciation events, including those associated with colour shift) or the survival of exotic morphs (high $\tau_{\max }$, or high $p_{d}$ ) decreases the effective speciation rate of species displaying a novel morph. Increased effective speciation rate - including speciation without colour shift - (when $\alpha_{\min }$, or $\tau_{\max }$ are low for instance) leads to a quick invasion of the grid, resulting into mosaics with lower numbers of patches. When allopatric speciation without colour shift is implemented, new morphs are more likely to persist because they are not in competition with their parental species, leading to more patchy mosaics $\left(s_{\text {symp }}=0.5\right.$, in Fig. S9).

\section{Temporal, geographical and ecological dynamics of species richness}

Both the probability of speciation associated with colour pattern shift $\left(s_{c}\right)$ and the number of possible morphs $\left(N_{\text {morph }}\right)$ affect the final species richness. Those parameters can alter directly the diversification rate. Moreover, they can affect indirectly the dynamics of species richness through their effects on the formation of the spatial mosaic of morphs.

- The proportion of speciation with colour shift $\left(s_{c}\right)$ and the number of possible morphs $\left(\mathrm{N}_{\text {morph }}\right)$ have direct and indirect effects on species richness.

As illustrated in Fig. 1, simulations with high values of $s_{c}$ or $N_{\text {morph }}$ lead to richer clades (Fig. 3A; the regression coefficients associated with ' $s_{c}$ ' and ' $N_{\text {morph }}$ ' are 25.30 and 0.32 respectively, with both Pvalue $<0.001$,

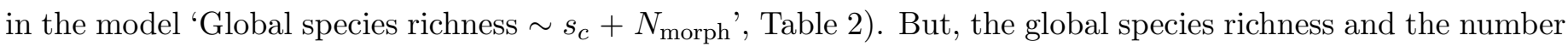
of patches of the resulting mosaic are highly correlated (Fig. 3A; the regression coefficient associated with 'Number of patches' is 19.96, Pvalue $<0.001$, in the model 'Global species richness $\sim s_{c}+N_{\text {morph }}+$ Number of patches', Table 2). If we control for its effects on the number of patches of the mosaics, increasing $s_{c}$ actually decreases the number of lineages persisting at the end (the regression coefficient associated with ' $s_{c}$ ' is -9.33 , 
Pvalue $<0.001$, in the model 'Global species richness $\sim s_{c}+N_{\text {morph }}+$ Number of patches', Table 2) because the effective speciation rate of species with exotic morphs is low due to positive frequency-dependent predation. Similarly, $N_{\text {morph }}$ does not significantly affect the diversification process if we control for its effect on the mosaic

(Pvalue $=0.99$ for the regression coefficient associated with ' $N_{\text {morph }}$ ', in the model 'Global species richness $\sim$ $s_{c}+N_{\text {morph }}+$ Number of patches', Table 2).

\section{- The formation of the spatial mosaic constrains species ranges and extends niche space.}

The formation of the spatial mosaic of morph leads to increased local and global species richnesses (Fig. 3A and $3 \mathrm{~B}$ ) for the following reasons. First, non-mimetic species occupying distant ecological niches in different patches can co-occur within a cell when speciation with colour shift leads to convergence toward the same colour pattern (Fig. 3E), increasing local species richness. Second, the emergence of a mosaic restricts species' range sizes (Fig. 3C) because species with a given morph have reduced probability to invade cells occupied by species displaying a different colour pattern. Such spatial segregation favours the maintenance of species occupying close ecological niches in different regions of the grid that would not have coexisted within cell because of inter-specific competition. Altogether, the formation of a patchy mosaic favours a more uniform occupation of the ecological niche space (Hartigans' dip statistic close to 0, in Fig. 3D, and see some examples of simulations in Fig. 1). It suggests that the local dominant colour pattern corresponds to a new dimension in the ecological niche space, allowing the same spatial grid to maintain more species. We note that simulations that did not lead to the formation of a spatial mosaic do not differ in their macroevolutionary patterns from simulations without mutualistic mimicry (i.e. without predation).

\section{- Many parameters have direct and indirect effects on species richness.}

All parameters affecting the number of patches of the resulting mosaic $\left(p_{d}, p_{s}, s_{\mathrm{symp}}, \tau_{\max }, \alpha_{\min }\right.$, curvature of the competition function in Fig. S10, S11, S7, S12, S13 and S14 respectively), thus alter the macroevolutionary pattern of species diversification. They also have direct effect on the dynamics of species richness. For instance, decreasing the minimum competition term possible $\left(\alpha_{\min }\right)$ or increasing the overall probability of speciation $\left(p_{s}\right)$ increases local species richness and therefore the global species richness (Fig. 5A and 5B). Similarly to $s_{c}$, the direct and the indirect effects of $\alpha_{\min }$ are antagonistic (low $\alpha_{\min }$ results in mosaics with low numbers of patches, but favours a high local species richness).

Overall, local selection mediated by mimicry can increase species diversification within clade, but mainly through the formation of multiple homogeneous patches shared by different species that display the same mimetic signal (see the summary diagram, Fig. 4A). 


\section{Phylogenetic tree shape and mimicry convergence}

The shape of reconstructed phylogenetic trees depends on the combination of parameters $s_{c}$ and $N_{\text {morph }}$, because of their effects on the diversification rate and on the emergence of the spatial mosaic of mimetic colour patterns.

\section{- The formation of a spatial mosaic balances the phylogenetic tree.}

The negative correlation observed between ' $N_{\text {morph' }}$ ' and the tree imbalance $I_{\mathrm{C}}$ (regression coefficient of $-2.60 \mathrm{e}-03$, with Pvalue $=0.04$ in the model ' $I_{\mathrm{C}} \sim s_{c}+N_{\text {morph }}$ ', Table 3 ) is driven by the effect of $N_{\text {morph }}$ on the formation of the spatial mosaic of morphs. Indeed, phylogenetic trees are significantly more balanced (lower $I_{\mathrm{C}}$ ) when mosaics are patchy (Fig. 3F; the regression coefficient associated with 'Number of patches' is $-7.30 \mathrm{e}-02$, Pvalue $<0.001$, in the model ' $I_{\mathrm{C}} \sim s_{c}+N_{\text {morph }}+$ Number of patches', Table 3). Species with large range sizes have more opportunities to give rise to new species in our model. Therefore, by balancing species range sizes, spatial segregation of species due to Müllerian mimicry gives similar opportunities of speciation to each lineage and leads to more balanced phylogenetic trees. Therefore, $N_{\text {morph }}$ favours tree balance indirectly, through its effect on the number of patches of the resulting mosaic.

- The formation of the spatial mosaic does not change the temporal dynamic of diversification.

A slowdown of the diversification rate through time $(\rho<0)$ is observed for all combinations of parameters tested (Fig. 3G): when ecological niche space fills, the effective speciation rate is reduced due to inter-specific competition. The statistic measuring temporal shift in diversification rate $\rho$ is positively correlated to $s_{c}$ and $N_{\text {morph }}$ (the regression coefficients associated with ' $s_{c}$ ' and ' $N_{\text {morph' }}$ ' are 0.14 and $2.52 \mathrm{e}-03$ respectively, with

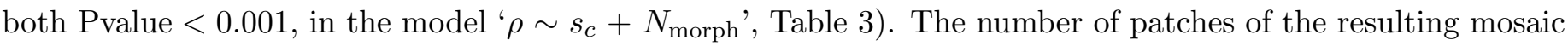
does not change the temporal dynamics of diversification (Pvalue $=0.10$ for the regression coefficient associate to 'Number of patches', in the model ' $\rho \sim s_{c}+N_{\text {morph }}+$ Number of patches', Table 3). Low values of $s_{c}$ or $N_{\text {morph }}$ lead to quicker occupation of the niche space by initially increasing the effective speciation rate. Thus, branching events slow down more rapidly as lineages accumulate, which results in nodes being more concentrated close to the root of the phylogeny (low $\rho$ values).

\section{- The proportion of convergence events observed depends on the spatial mosaic.}

The proportion of speciation events with colour shift $s_{c}$ and the probability of convergence to an existing morph $\left(1 / N_{\text {morph }}\right)$ both favour the occurrence of convergence events (Fig. 3E; the regression coefficients associated with ' $s_{c}$ ' and ' $N_{\text {morph' }}$ ' are $9.41 \mathrm{e}-02$ and $-1.03 \mathrm{e}-03$, with both Pvalue $<0.001$, in the model 'Proportion of convergence events $\sim s_{c}+N_{\text {morph }}$ ', Table 3$)$. We still observe this effect when we control for the number of patches of the mosaics (the regression coefficients associated with ' $s_{c}$ ' and ' $N_{\text {morph }}$ ' are $8.40 \mathrm{e}-02$ 
and $-1.24 \mathrm{e}-03$, Pvalue $<0.001$, in the model 'Proportion of convergence events $\sim s_{c}+N_{\text {morph }}+$ Number of patches', Table 3). However, the number of patches of the mosaic is positively correlated with the proportion of convergence events (the regression coefficient associated with 'Number of patches' is 1.48e - 02, Pvalue $<0.001$, in the same linear model, Table 3 ). Therefore, $s_{c}$ and $N_{\text {morph }}$ affect directly and indirectly (through their effect on the formation of the mosaic) the proportion of convergence events observed. In particular, as expected, a high probability of convergence (incurred by a low $N_{\text {morph }}$ ) has a positive direct effect on the proportion of convergence events observed in the reconstructed phylogeny. But its indirect effect is negative: a high probability of convergence favours the formation of mosaics with a low number of patches, giving us little opportunity to observe convergence events.

\section{- Many parameters have direct and indirect effects on the phylogenetic tree shape and on} convergence.

The effects of other parameters on tree imbalance are mainly mediated by their effect on the spatial distribution of morphs. Indeed, the parameters $s_{\text {symp }}$ (Fig. S7), $p_{d}$ (Fig. S10), $p_{s}$ (Fig. S11), $\tau_{\max }$ (Fig. S12), $\alpha_{\min }$ (Fig. S13) affect the number of patches of the resulting mosaic, which alters the imbalance of the resulting phylogenies (Fig. 5). They have also indirect and direct effects on the proportion of convergence events. Those effects can be redundant (high $s_{\text {symp }}$, high $p_{d}$ and low $\alpha_{\min }$ result in mosaics with a low number of patches, and directly favour a low proportion of convergence events) or contradictory (low $p_{s}$ results in mosaics with a low number of patches, but favours a high proportion of convergence events).

Therefore, the emergence of the spatial mosaic greatly affects the shape of phylogenies of mimetic organisms (see the summary diagram, Fig. 4B, 4C and 4D). Müllerian mimicry is a mutualistic interaction, which is expected to lead to more balanced trees due to spatial segregation. Our model also predicts that mimetic clades where numerous convergence events are observed are not necessarily those with the highest probabilities of convergence. High probability of convergence leads to mosaics with a low number of patches in a context of invasion, and therefore allows little opportunity to actually observe these convergence events in the resulting phylogeny. 


\section{DISCUSSION}

Our model demonstrates that spatial variation of the mimetic environment should affect the diversification process at a macroevolutionary scale. First, constraints imposed by ecological interactions on species range size can equalize speciation rates among lineages, resulting in more balanced phylogenies. Secondly, mutualistic coevolutionary dynamics can promote a larger coverage of the available ecological niche space, thereby increasing the overall species richness in the clade.

\section{Ecological interactions constrain species range size and balance speciation rates among lineages}

Phylogenies can be analyzed to investigate the underlying processes of diversification. To extract such information from empirical phylogenies, many studies have focused on dissecting how abiotic and biotic factors affect the shape of phylogenies. Here we provide theoretical evidence that mimetic interactions can balance the resulting phylogenies through constraints they exert on species geographic distribution. Since ecological speciation is a local process, we implemented a constant probability of ecological speciation per cell. As a consequence, lineages with large range size - i.e. covering a large number of cells - have higher speciation rates. Thus, this assumption of our model promotes differences in speciation rate between species with small vs. large range size. If there are no spatial constraints, a small number of species with large range size give rise to large number of derived species, leading to unbalanced trees. Geographical constraints imposed by Müllerian mimicry reduce the differences between species range sizes, thereby homogenizing speciation rates among lineages. Hence, the resulting phylogenies are more balanced when the spatial mosaics of morphs are patchy.

Empirical investigations (1) testing whether mimetic clades are more balanced than non-mimetic clades or (2) assessing the correlation between patchiness and tree balance among mimetic clades or subclades are needed to estimate the actual effects of mimetic interactions on diversification over large phylogenetic scale. However, in order to test the different predictions proposed in our study, information about species spatial distributions (including subspecies distributions if some exhibit different mimetic morphs), phylogeny and colour patterns are needed. Clades that fulfill these three conditions remain currently rare. Yet, we hope our theoretical results will stimulate further research in that direction. Moreover, we expect similar constraints on range size between species involved in other forms of obligate mutualism, where mutualistic partners are completely interdependent for survival (e.g. symbiotic relationship between algae and fungus in lichens). We thus encourage testing our theoretical predictions on empirical data in organisms involved in such mutualistic interactions.

Mimetic species often harbor multiple geographic races displaying distinct colour patterns (Mallet and Joron 1999). Polymorphic species gain protection against predators in different geographical areas by displaying a locally-abundant colour pattern. Polymorphic species' range sizes are therefore not limited by the spatial mosaic, reducing the indirect effect of colour shift during speciation at macroevolutionary scale. For simplicity, we did 
not consider polymorphic species in the model. Given the direct influence of colour pattern shift on speciation rate, these races might correspond to early stages in the ecological speciation continuum (Chamberlain et al. 2009) [but see Martin et al. (2013)], and can therefore be regarded as incipient species. We also did not observe the maintenance of different warning colour patterns within cells because of the strong frequency-dependent selection implemented in our model. However, local colour pattern diversity is readily observed in mimetic communities of neotropical ithomiine (Beccaloni 1997) and heliconiine butterflies (Rosser et al. 2015), leaf beetles (Borer et al. 2010) or frogs (Chouteau and Angers 2012), due to correlated microhabitat segregation for predators and prey within communities (Elias et al. 2008; Gompert et al. 2011). Here, we did not implement microhabitat segregation, because this would amount to subdivide each cell into smaller items (microhabitats), and would lead to similar results, but at a smaller scale.

Other biotic interactions such as competition, reproductive interference or predation are known to generate and maintain parapatric distributions (Bull 1991); therefore they could have effects on macroevolutionary patterns similar to those driven by mutualistic mimicry. Moen and Morlon (2014) already suggested that antagonistic ecological interactions could indirectly affect macroevolutionary patterns of diversification when considering the influence of geography on diversification rates. As highlighted here, other features of speciation (geographic mode of speciation, probability of speciation, ecological similarity between species) and other processes (local catastrophes) can favour the formation of spatial mosaics of morphs, and have therefore an indirect effect on macroevolutionary patterns. This stresses the need for theoretical and empirical studies exploring direct and indirect effects of any ecological process constraining species range size.

Finally, indirect effects of ecological interactions on the shape of phylogenies may depend on the mode of speciation considered (i.e. allopatric vs. ecological speciation). In our model, allowing a fraction of the speciation events to be allopatric facilitates the formation of patchy mosaics and therefore leads to richer and more balanced clades than when speciation is only sympatric. This suggests that allopatric speciation may be of prime importance to explain the diversification of mimetic clades and the way they are spatially structured. Landscapes may change because of the formation of mountains or islands. Changes in climatic conditions, which can isolate population and drive allopatric speciation, may also be largely responsible for the mimetic mosaics that are commonly observed (Brown et al. 1974; Brower 1996). For simplicity, we assumed that allopatric speciation occurs within a single cell, with equal probability in each cell. However, speciation due to vicariance is likely to affect species in multiple cells and its probability may be higher for species with intermediate range size (Pigot et al. 2010). Indeed, even if there is a high probability that a geographical barrier affects species with large range sizes, this geographical barrier may not go through the entire range. Therefore, when species range size is constrained by ecological interactions, the balance of the phylogeny and the temporal shift in diversification rate may be different under a model of vicariant speciation where barriers can affect multiple 
cells.

\section{Mutualistic mimicry extends the number of ecological niche dimensions and enhances diversifi- cation through the formation of the spatial mosaic of morphs}

Mimetic colour patterns have been suggested to be "magic traits" - i.e. traits under divergent selection that also contribute to non-random mating (Servedio et al. 2011) - that drive speciation (Jiggins 2008). We did not implement such direct effects of colour pattern shift on the probability of speciation, which makes our model conservative with respect to the effect of colour pattern shifts on diversification of mimetic clades. However, under such conditions and despite positive frequency-dependent selection against non-mimetic derived species, we showed that implementing speciation with colour pattern shift greatly enhances diversification of Müllerian mimetic clades. The enhanced diversification of mimetic clades is entirely attributable to the formation of spatial mosaics, since increasing the proportion of speciation events with a colour pattern shift after controlling for the number of patches actually decreases species richness in our simulations. Observations of high diversification rates in Müllerian mimetic clades are in agreement with this prediction. A recent empirical study on amphibians indeed showed that clades exhibiting chemical defenses and warning colour patterns are characterized by increased diversification rates (Arbuckle and Speed 2015). Similarly, the neotropical mimetic butterflies tribes Heliconiini (Nymphalidae: Heliconiinae, 100 species) and Ithomiini (Nymphalidae: Danainae, 390 species) are remarkable by their species richness. Müllerian mimicry might be viewed as a key-innovation, the acquisition of which is repeatedly accompanied by an increased diversification rate due to the emergence of spatial variation of the mimetic environment.

We showed that this spatial segregation of mimetic communities favours a more uniform occupation of the ecological niche space, and limits the diversification slowdown triggered by ecological niche filling. Under Hutchinson's definition, ecological niche coordinates describe the environmental conditions (temperature, available resources...) necessary for a species to persist (Hutchinson 1957). Ecological niche filling has been hypothesized to slow diversification down (Mayr 1942, 1947; Simpson 1953; Phillimore and Price 2008; Rabosky and Lovette 2008). By explicitly modelling the ecological niche space, we do observe diversification slowdown due to niche filling in our simulated phylogenetic trees, but this slowdown is delayed in cases where a spatial mosaic of morphs emerges. Because of positive frequency-dependent selection pressure exerted by predators, individuals exhibiting the locally dominant morph benefit from a survival advantage. The local mimetic pattern can therefore be considered as a new dimension of the niche, because species can persist in a locality only if they display the dominant colour pattern, i.e. the pattern most likely to be avoided by the predator community. In the cases where a spatial mosaic is formed, this new niche dimension allows two ecologically similar species that should exclude one another to persist if they harbor different colour pattern, because they are spatially seg- 
regated. Therefore, geographical variation of mimicry ring composition indirectly enhances the diversification process by increasing the ecological niche space available. Contrary to the two other niche dimensions initially implemented, mimetic colour pattern represents a dimension of the ecological niche that generates a positive interaction between species sharing the same colour pattern. On the other hand, it generates an antagonistic interaction between co-occurring species with different colour patterns, because species with less abundant colour patterns are eliminated by predation, due to positive frequency-dependent selection.

Our study highlights that mutualistic coevolution - mediated by Müllerian mimicry - can be geographically structured, and that such spatial variation of the biotic environment corresponds to multiple ecological niches. Empirical studies have provided support for the existence of similar mosaic when other mutualistic interactions are involved, such as legume-rhizobial symbioses (Parker 1999), mycorrhizal symbioses (Johnson et al. 2010) or plant-pollinator mutualism (Anderson and Johnson 2008). Defense mutualism - the ability of plants to form mutualistic interactions with animal defenders - has already been hypothesized to expand the realized niche of a plant (Bruno et al. 2003) and to favour diversification, by increasing the probability that a lineage will encounter conditions with ecological opportunity (Weber and Agrawal 2014). More generally, many forms of coevolution generate geographic constraints by imposing tight links between interacting species (see the Geographic Mosaic Theory of Coevolution synthesized in Thompson 2005). Antagonistic interactions, between competitors for resources (Benkman et al. 2001), host-parasites (Burdon and Thrall 1999; Nuismer et al. 2003; Lively et al. 2004) or between prey and predators (Toju and Sota 2006; Brodie Jr et al. 2002), are recognized to promote spatial variation of coevolution. Due to the formation and the maintenance of the geographic mosaic of coevolution - driven by random genetic drift for instance (Kiester et al. 1984; Sherratt 2006) - the number of niche dimensions should be higher. Thus, clades involved not only in antagonistic coevolution but also in mutualistic coevolution are expected to be more diverse.

\section{CONCLUSION AND PERSPECTIVES}

Our theoretical work suggests that Müllerian mimicry, and possibly other forms of obligate mutualistic interactions, can favour the diversification process at a macroevolutionary scale by constraining species range sizes and by extending the ecological niche space. Only a handful of empirical studies have shown that coevolution in mutualisms can result in stable mosaic patterns of spatial differentiation, perhaps because theoretical conditions leading to the formation of such mosaics are limited. In the well-documented example of mutualistic Müllerian mimicry, multiple factors generating colour pattern diversity have been identified, although polymorphism in Müllerian mimicry has been considered paradoxical for decades. We now need to understand to what extent other forms of mutualism can lead to the formation and the maintenance of mosaics of coevolution. It would help to understand large-scale patterns of diversification, since we showed that such mosaic of coevolution is expected 
to promote species-rich clades and to balance phylogenetic trees. Our theoretical approach also stresses the need to empirically investigate the link between spatial mosaics of coevolution and macroevolutionary patterns. It could guide future empirical research in Müllerian mimetic clades by providing clear causal relationships between local processes and macroevolutionary patterns of diversification. Overall, our theoretical simulations are shedding light on the conditions under which macroevolutionary patterns of diversification have emerged in nature.

\section{Acknowledgments}

We thank J.H. Pantel and T.N. Sherratt for their comments on manuscript drafts. We are very grateful to two anonymous reviewers for comments and suggestions that improved our manuscript. Simulations were run with the computer cluster 'Plateforme Calcul Intensif Algorithmique' (UMS2700-PCIA) of the MNHN. We are very grateful to J. Pedraza for his help with the cluster. T.G.A. was funded by a bursary and a PhD scholarship from the French Ministry of Higher Education and Research. M.E. acknowledges support from an ATIP grant and from a research grant from the French National Agency for Research (SPECREP). V.L. acknowledges support from a young research grant from the French National Agency for Research (ANR-13-JSV7-0003-01-Domevol) and an Emergence grant from Mairie de Paris. N.C. was funded by a PhD scholarship from the French Ministry of Higher Education and Research and a postdoc grant of the MNHN. 


\section{References}

Agapow, P. M. and A. Purvis, 2002. Power of eight tree shape statistics to detect nonrandom diversification: a comparison by simulation of two models of cladogenesis. Systematic Biology 51:866-872.

Alexandrou, M. A., C. Oliveira, M. Maillard, R. A. R. McGill, J. Newton, S. Creer, and M. I. Taylor, 2011. Competition and phylogeny determine community structure in Müllerian co-mimics. Nature 469:84-88.

Althoff, D. M., K. A. Segraves, and M. T. J. Johnson, 2014. Testing for coevolutionary diversification: linking pattern with process. Trends in Ecology and Evolution 29:82-89.

Anderson, B. and S. D. Johnson, 2008. The Geographical mosaic of coevolution in a plant-pollinator mutualism. Evolution 62:220225.

Arbuckle, K. and M. P. Speed, 2015. Antipredator defenses predict diversification rates. Proceedings of the National Academy of Sciences of the United States of America 112:13597-13602.

Aubier, T. G. and T. N. Sherratt, 2015. Diversity in Müllerian mimicry: the optimal predator sampling strategy explains both local and regional polymorphism in prey. Evolution 69:2831-2845.

Aubier, T. G., M. Joron, and T. N. Sherratt, in press. Mimicry among unequally defended prey should be mutualistic when predators sample optimally. The American Naturalist.

Beatty, C. D., K. Beirinckx, and T. N. Sherratt, 2004. The evolution of müllerian mimicry in multispecies communities. Nature 431:63-66.

Beccaloni, G. W, 1997. Ecology, natural history and behaviour of ithomiine butterflies and their mimics in Ecuador (Lepidoptera: Nymphalidae: Ithomiinae). Tropical Lepidoptera 8:103-124.

Benkman, C. W., W. C. Holimon, and J. W. Smith, 2001. The influence of a competitor on the geographic mosaic of coevolution between crossbills and lodgepole pine. Evolution 55:282-294.

Birand, A., A. Vose, and S. Gavrilets, 2012. Patterns of species ranges, speciation, and extinction. The American Naturalist $179: 1-21$.

Blum, M. G. B., O. François, and S. Janson, 2006. The mean, variance and limiting distribution of two statistics sensitive to phylogenetic tree balance. The Annals of Applied Probability 16:2195-2214.

Bolnick, D., 2004. Can intraspecific competition drive disruptive selection? An experimental test in natural populations of sticklebacks. Evolution 58:608-618.

Borer, M., T. Van Noort, M. Rahier, and R. E. Naisbit, 2010. Positive frequency-dependent selection on warning color in alpine leaf beetles. Evolution 64:3629-3633.

Brodie, E. D. Jr., B. J. Ridenhour, and E. D. Brodie III, 2002. The evolutionary response of predators to dangerous prey: hotspots and coldspots in the geographic mosaic of coevolution between garter snakes and newts. Evolution 56:2067-2082.

Brower, A. V. Z., 1996. Parallel race formation and the evolution of mimicry in Heliconius butterflies: a phylogenetic hypothesis from mitochondrial DNA sequences. Evolution 50:195-221.

Brown, K. S., P. M. Sheppard, and J. R. G. Turner, 2003. Quaternary refugia in tropical America: evidence from race formation in Heliconius butterflies. Proceedings of the Royal Society of London. Series B, Biological Sciences 187:369-378.

Bruno, J. F., J. J. Stachowicz, and M. D. Bertness, 2003. Inclusion of facilitation into ecological theory. Trends in Ecology and Evolution 18:119-125.

Bull, C. M., 1991. Ecology of parapatric distributions. Annual Review of Ecology and Systematics 22:19-36.

Burdon, J. J. and P. H. Thrall, 1999. Spatial and temporal patterns in coevolving plant and phatogen associations. The American Naturalist 153:S15-S33.

Cavender-Bares, J., K. H. Kozak, P. V. A. Fine, and S. W. Kembel, 2009. The merging of community ecology and phylogenetic biology. Ecology Letters 12:693-715.

Chamberlain, N. L., R. I. Hill, D. D. Kapan, L. E. Gilbert, and M. R. Kronforst, 2009. Polymorphic butterfly reveals the missing link in ecological speciation. Science 326:847-850. 
Chazot, N., K. R. Willmott, P. G. Santacruz Endara, A. Toporov, R. I. Hill, C. D. Jiggins, and M. Elias, 2014. Mutualistic mimicry and filtering by altitude shape the structure of Andean butterfly communities. The American Naturalist 183:26-39.

Chouteau, M. and B. Angers, 2011. The role of predators in maintaining the geographic organization of aposematic signals. The American Naturalist 178:810-817.

—

Chouteau, M., M. Arias, and M. Joron, 2016. Warning signals are under positive frequency-dependent selection in nature. Proceedings of the National Academy of Sciences of the United States of America 113:2164-2169.

Colless, D. H., 1982. [Review of] Phylogenetics: the theory and practice of phylogenetic systematics. Systematic Zoology 31:100-104.

Darwin, C., 1871. The Descent of Man, and Selection in Relation to Sex. Princeton University Press.

Dieckmann, U. and M. Doebeli, 1999. On the origin of species by sympatric speciation. Nature 400:354-357.

Dumbacher, J. P. and R. C. Fleischer, 2001. Phylogenetic evidence for colour pattern convergence in toxic pitohuis: Müllerian mimicry in birds? Proceedings of the Royal Society B: Biological Sciences 268:1971-1976.

Ehrlich, P. R. and P. H. Raven, 1964. Butterflies and Plants: A Study in Coevolution. Evolution 18:586-608.

Elias, M., Z. Gompert, C. Jiggins, and K. Willmott, 2008. Mutualistic interactions drive ecological niche convergence in a diverse butterfly community. PLoS Biology 6:2642-2649.

Erwin, D. H., 2000. Macroevolution is more than repeated rounds of microevolution. Evolution and Development 2:78-84.

— 2009. Climate as a driver of evolutionary change. Current Biology 19:R575-R583.

Figueirido, B., C. M. Janis, J. A. Pérez-Claros, M. De Renzi, and P. Palmqvist, 2012. Cenozoic climate change influences mammalian evolutionary dynamics. Proceedings of the National Academy of Sciences of the United States of America 109:722-727.

Franks, D. W., G. D. Ruxton, and T. N. Sherratt, 2008. Warning signals evolve to disengage Batesian mimics. Evolution 63:256-267.

Fraser, D., R. Gorelick, and N. Rybczynski, 2015. Macroevolution and climate change influence phylogenetic community assembly of North American hoofed mammals. Biological Journal of the Linnean Society 114:485-494.

Freeman, J. B. and R. Dale, 2013. Assessing bimodality to detect the presence of a dual cognitive process. Behavior Research Methods 45:83-97.

Gómez, J. M. and M. Verdú, 2012. Mutualism with plants drives primate diversification. Systematic Biology 61:567-577.

Gompert, Z., K. Willmott, and M. Elias, 2011. Heterogeneity in predator micro-habitat use and the maintenance of Müllerian mimetic diversity. Journal of Theoretical Biology 281:39-46.

Grant, P. R. and B. R. Grant, 2006. Evolution of character displacement in Darwin's finches. Science 313:224-226.

Hartigan, J. A. and P. M. Hartigan, 1985. The dip test of unimodality. The Annals of Statistics 13:70-84.

Hembry, D. H., J. B. Yoder, and K. R. Goodman, 2014. Coevolution and the diversification of life. The American Naturalist 184:425-438.

Hodges, S. A., 1997. Floral nectar spurs and diversification. International Journal of Plant Sciences 158:S81-S88.

Hutchinson, G. E., 1957. Concluding remarks. Cold Spring Harbor Symposia on Quantitative Biology 22:415-427.

Jablonski, D., 2007. Scale and hierarchy in macroevolution. Palaeontology 50:87-109.

— 2008a. Biotic interactions and macroevolution: extensions and mismatches across scales and levels. Evolution 62:715-739.

- 2008b. Colloquium paper: extinction and the spatial dynamics of biodiversity. Proceedings of the National Academy of Sciences of the United States of America 105:11528-11535.

Jablonski, D. and K. Roy, 2003. Geographical range and speciation in fossil and living molluscs. Proceedings of the Royal Society B: Biological Sciences 270:401-406.

Jiggins, C. D., 2008. Ecological speciation in mimetic butterflies. Bioscience 58:541-548. 
Jiggins, C. D., R. Mallarino, K. R. Willmott, and E. Bermingham, 2006. The phylogenetic pattern of speciation and wing pattern change in neotropical Ithomia butterflies (Lepidoptera: Nymphalidae). Evolution 60:1454-1466.

Jiggins, C. D., R. E. Naisbit, R. L. Coe, and J. Mallet, 2001. Reproductive isolation caused by colour pattern mimicry. Nature 411:302-305.

Johnson, N. C., G. W. T. Wilson, M. A. Bowker, J. A. Wilson, and R. M. Miller, 2010. Resource limitation is a driver of local adaptation in mycorrhizal symbioses. Proceedings of the National Academy of Sciences of the United States of America 107:2093-2098.

Joy, J. B., 2013. Symbiosis catalyses niche expansion and diversification. Proceedings of the Royal Society B: Biological Sciences 280:20122820.

Kapan, D. D., 2001. Three-butterfly system provides a field test of müllerian mimicry. Nature 409:338-340.

Kiessling, W. and M. Aberhan, 2007. Geographical distribution and extinction risk: lessons from Triassic-Jurassic marine benthic organisms. Journal of Biogeography 34:1473-1489.

Kiester, A. R., R. Lande, and D. W. Schemske, 1984. Models of coevolution and speciation in plants and their pollinators. The American Naturalist 124:220-243.

Kirkpatrick, M. and M. Slatkin, 1993. Searching for evolutionary patterns in the shape of a phylogenetic tree. Evolution 47:11711181.

Kopp, M. and S. Gavrilets, 2006. Multilocus genetics and the coevolution of quantitative traits. Evolution 60:1321-1336.

Law, R., P. Marrow, and U. Dieckmann, 1997. On evolution under asymmetric competition. Evolutionary Ecology 11:485-501.

Litsios, G., C. A. Sims, R. O. Wüest, P. B. Pearman, N. E. Zimmermann, and N. Salamin, 2012. Mutualism with sea anemones triggered the adaptive radiation of clownfishes. BMC Evolutionary Biology 12.

Lively, C. M., M. F. Dybdahl, J. Jokela, E. E. Osnas, and L. F. Delph, 2004. Host sex and local adaptation by parasites in a snail-trematode interaction. The American Naturalist 164:S6-S18.

Magallón, S. and M. J. Sanderson, 2001. Absolute diversification rates in angiosperm clades. Evolution 55:1762-1780.

Mallet, J., 2010. Shift happens! Shifting balance and the evolution of diversity in warning colour and mimicry. Ecological Entomology 35:90-104.

Mallet, J. and N. H. Barton, 1989. Strong natural selection in a warning-color hybrid zone. Evolution 43:421-431.

Mallet, J. and M. Joron, 1999. Evolution of diversity in warning color and mimicry: polymorphisms, shifting balance, and speciation. Annual Review of Ecology and Systematics 30:201-233.

Marek, P. E. and J. E. Bond, 2009. A Müllerian mimicry ring in Appalachian millipedes. Proceedings of the National Academy of Sciences of the United States of America 106:9755-9760.

Martin, S. H., K. K. Dasmahapatra, N. J. Nadeau, C. Salazar, J. R. Walters, F. Simpson, M. Blaxter, A. Manica, J. Mallet, and C. D. Jiggins, 2013. Genome-wide evidence for speciation with gene flow in Heliconius butterflies. Genome Research 23:1817-1828.

Mayr, E., 1942. Systematics and the Origin of Species. Columbia Univ. Press, New York.

- 1947. Ecological factors in speciation. Evolution 1:263-288.

McBride C. S. and M. C. Singer, 2010. Field studies reveal strong postmating isolation between ecologically divergent butterfly populations. PLoS Biology 8:e1000529.

McPeek, M. A., 2008. The ecological dynamics of clade diversification and community assembly. The American Naturalist 172:E270E284.

Merrill, R. M., B. Van Schooten, J. A. Scott, and C. D. Jiggins, 2011. Pervasive genetic associations between traits causing reproductive isolation in Heliconius butterflies. Proceedings of the Royal Society B: Biological Sciences 278:511-518.

Merrill, R. M., R. W. R. Wallbank, V. Bull, P. C. A. Salazar, J. Mallet, M. Stevens, and C. D. Jiggins, 2012. Disruptive ecological selection on a mating cue. Proceedings of the Royal Society B: Biological Sciences 279:4907-4913. 
Moen, D. and H. Morlon, 2014. Why does diversification slow down? Trends in Ecology and Evolution 29:190-197.

Müller, F., 1879. Ituna and Thyridia: a remarkable case of mimicry in butterflies. Proceedings of the Entomological Society of London 2:25-29.

Newell, N. D., 1952. Periodicity in Invertebrate Evolution. Journal of Paleontology 26:371-385.

Nuismer, S. L., J. N. Thompson, and R. Gomulkiewicz, 2003. Coevolution between hosts and parasites with partially overlapping geographic ranges. Journal of Evolutionary Biology 16:1337-1345.

Papageorgis, C., 1975. Mimicry in neotropical butterflies. American Scientist 63:522-532.

Parker, M. A., 1999. Mutualism in metapopulations of legumes and rhizobia. The American Naturalist 153:S48-S60.

Peters, S. E., 2005. Geologic constraints on the macroevolutionary history of marine animals. Proceedings of the National Academy of Sciences of the United States of America 102:12326-12331.

Phillimore, A. B. and T. D. Price, 2008. Density-dependent cladogenesis in birds. PLoS biology 6:483-489.

Pigot, A. L., A. B. Phillimore, I. P. F. Owens, and C. D. L. Orme, 2010. The shape and temporal dynamics of phylogenetic trees arising from geographic speciation. Systematic Biology 59:660-673.

Pigot, A. L. and J. A. Tobias, 2013. Species interactions constrain geographic range expansion over evolutionary time. Ecology Letters 16:330-338.

Pybus, O. G. and P. H. Harvey, 2000. Testing macroevolutionary models using incomplete molecular phylogenies. Proceedings of the Royal Society B: Biological Sciences 267:2267-2272.

Rabosky, D. L., 2013. Diversity-dependence, ecological speciation, and the role of competition in macroevolution. Annual Review of Ecology, Evolution, and Systematics 44:481-502.

Rabosky, D. L. and I. J. Lovette, 2008. Density-dependent diversification in North American wood warblers. Proceedings of the Royal Society B: Biological Sciences 275:2363-2371.

Ranjard, L., D. Welch, M. Paturel, and S. Guindon, 2014. Modelling competition and dispersal in a statistical phylogeographic framework. Systematic Biology 63:743-752.

Ricklefs, R. E., 2010. Evolutionary diversification, coevolution between populations and their antagonists, and the filling of niche space. Proceedings of the National Academy of Sciences of the United States of America 107:1265-1272.

Rosindell, J., S. J. Cornell, S. P. Hubbell, and R. S. Etienne, 2010. Protracted speciation revitalizes the neutral theory of biodiversity. Ecology Letters 13:716-727.

Rosser, N., K. M. Kozak, A. B. Phillimore, and J. Mallet, 2015. Extensive range overlap between heliconiine sister species: evidence for sympatric speciation in butterflies? BMC Evolutionary Biology 15:125.

Rowland, H. M., E. Wiley, G. D. Ruxton, J. Mappes, and M. P. Speed, 2010b. When more is less: the fitness consequences of predators attacking more unpalatable prey when more are presented. Biology Letters 6:732-735.

Sargent, R. D., 2004. Floral symmetry affects speciation rates in angiosperms. Proceedings of the Royal Society B: Biological Sciences 271:603-608.

Sbordoni, V., L. Bullini, G. Scarpellii, S. Forestiero, and M. Rampini, 1979. Mimicry in the burnet moth Zygaena ephialtes: population studies and evidence of a Batesian-Müllerian situation. Ecological Entomology 4:83-93.

Schluter, D., 1994. Experimental evidence that competition promotes divergence in adaptive radiation. Science 266:798-801.

Sepkoski, J. J., 1978. A kinetic model of Phanerozoic taxonomic diversity I. Analysis of marine orders. Paleobiology 4:223-251.

Sheppard, P. M., J. R. G. Turner, K. S. Brown, W. W. Benson, and M. C. Singer, 1985. Genetics and the evolution of Muellerian mimicry in Heliconius butterflies. Philosophical Transactions of the Royal Society: Biological Sciences 308:433-610.

Sherratt, T. N., 2006. Spatial mosaic formation through frequency-dependent selection in Müllerian mimicry complexes. Journal of Theoretical Biology 240:165-174.

Servedio, M. R., G. S. Van Doorn, M. Kopp, A. M. Frame, and P. Nosil, 2011. Magic traits in speciation: 'magic' but not rare?. Trends in Ecology and Evolution 26:389-397. 
Simpson, G. G., 1953. The Major Features of Evolution. Columbia University Press.

Symula, R., R. Schulte, and K. Summers, 2001. Molecular phylogenetic evidence for a mimetic radiation in Peruvian poison frogs supports a Müllerian mimicry hypothesis. Proceedings of the Royal Society B: Biological Sciences 268:2415-2421.

Thompson, J. N., 2005. The Geographic Mosaic of Coevolution. University Of Chicago Press.

Toju, H. and T. Sota, 2006. Imbalance of predator and prey armament: geographic clines in phenotypic interface and natural selection. The American Naturalist 167:105-117.

Weber, M. G. and A. A. Agrawal, 2014. Defense mutualisms enhance plant diversification. Proceedings of the National Academy of Sciences of the United States of America 111:16442-16447.

Weiner, J., 1990. Asymmetric competition in plant populations. Trends in Ecology and Evolution 5:360-364.

Williams, P., 2007. The distribution of bumblebee colour patterns worldwide: possible significance for thermoregulation, crypsis, and warning mimicry. Biological Journal of the Linnean Society 92:97-118.

Willmott, K. R. and J. Mallet, 2004. Correlations between adult mimicry and larval host plants in ithomiine butterflies. Proceedings of the Royal Society B: Biological Sciences 271:S266-S269.

Wilson, J. S., K. a. Williams, M. L. Forister, C. D. von Dohlen, and J. P. Pitts, 2012. Repeated evolution in overlapping mimicry rings among North American velvet ants. Nature Communications 3:1272.

Yoder, J. B. and S. L. Nuismer, 2010. When does coevolution promote diversification? The American Naturalist 176:802-17. 


\section{TABLES}

Table 1: Notations and numerical values.

\begin{tabular}{lll}
\hline Evolving trait & Definition & Range \\
\hline$m_{i}$ & Morph of species $i$ & $m_{i} \in\left\{1, \ldots N_{\text {morph }}\right\}$ \\
$z_{i}=\left(x_{i}, y_{i}\right)$ & Niche occupied by species $i$ : pair of ecological traits $x_{i}, y_{i}$ & Default value \\
\hline Parameter & Definition & 0.2 \\
\hline$\alpha_{\text {min }}$ & Minimum competition term possible & 4 \\
$b$ & Number of offspring per individual & 500 \\
$C$ & Correlation coefficient linking the niche distance $d_{i j}{ }^{f}$ to $\alpha_{i, j}$ & 5 \\
$f$ & Factor of the polynomial relationship between $\alpha_{i j}$ and $d_{i j}$ & 20 \\
$G$ & Size of the grid $G \times G$ & 50 \\
$K$ & Species carrying capacity in each cell & 0.001 \\
$\mu$ & Migration rate & {$[4,30]$} \\
$N_{\text {morph }}$ & Number of possible morphs & $1.10^{-5}$ \\
$p_{d}$ & Probability of cell destruction at any time step & 0.9 \\
$p_{p}$ & Probability of predator presence in a cell at any time step & $2.10^{-5}$ \\
$p_{s}$ & Probability of speciation of each species in a cell at any time step & $\{0,0.25,0.50,0.75\}$ \\
$s_{c}$ & Proportion of speciation events with colour shift & $1-s_{c}$ \\
$s_{n}$ & Proportion of speciation events without colour shift & 1 \\
$s_{\text {symp }}$ & Proportion of speciation events in sympatry & 0.05 \\
$\sigma_{N}$ & Standard deviation of niche divergence & 10 \\
$\tau_{\max }$ & Number of prey attacked before predation on a given morph ceases & \\
\hline
\end{tabular}


Table 2: Multivariate linear regression model. Species richness, range size and ecology.

\begin{tabular}{ccccccccc}
\hline \multirow{2}{*}{ Term } & \multicolumn{2}{c}{ Global species richness } & \multicolumn{2}{c}{ Local species richness } & \multicolumn{2}{c}{ Median of species range size } & \multicolumn{2}{c}{ Hartigans' dip statistic } \\
\cline { 2 - 9 } & Estimate & Pvalue & Estimate & Pvalue & Estimate & Pvalue & Estimate & Pvalue \\
\hline$s_{c}$ & $\mathbf{2 5 . 3 0}$ & $<0.001$ & $\mathbf{- 0 . 8 3}$ & $<0.001$ & $\mathbf{- 1 9 8 . 9 8}$ & $<0.001$ & $\mathbf{- 3 . 4 3 e - 0 2}$ & $<0.001$ \\
$N_{\text {morph }}$ & $\mathbf{0 . 3 2}$ & $<0.001$ & $\mathbf{1 . 4 4 e - 0 2}$ & 0.003 & $\mathbf{- 1 . 1 5}$ & $<0.001$ & $\mathbf{- 3 . 4 7 e - 0 4}$ & $<0.001$ \\
\hline$s_{c}$ & $\mathbf{- 9 . 3 3}$ & $<0.001$ & $\mathbf{- 2 . 8 5}$ & $<0.001$ & $\mathbf{- 5 3 . 7 6}$ & $<0.001$ & $\mathbf{9 . 7 4 e - 0 3}$ & $<0.001$ \\
$N_{\text {morph }}$ & $-6.69 \mathrm{e}-5$ & 0.99 & $-4.51 \mathrm{e}-03$ & 0.31 & 0.20 & 0.33 & $\mathbf{7 . 1 0 e - 0 5}$ & 0.22 \\
Number of patches & $\mathbf{1 9 . 9 6}$ & $<0.001$ & $\mathbf{1 . 1 6}$ & $<0.001$ & $\mathbf{- 8 3 . 7 5}$ & $<0.001$ & $\mathbf{- 2 . 5 2 e - 0 2}$ & $<0.001$ \\
\hline
\end{tabular}

Note: Multivariate linear models (least-square estimation) : Term $\sim s_{c}+N_{\text {morph }}$, and Term $\sim s_{c}+N_{\text {morph }}+$ Number of patches. Estimates that are significantly different than 0 are in bold (Pvalue of the t-test $<0.05$ ).

Table 3: Multivariate linear regression model. Mimicry convergence and shape of phylogenies.

\begin{tabular}{ccccccc}
\hline \multirow{2}{*}{ Term } & \multicolumn{2}{c}{ Proportion of } & \multicolumn{2}{c}{} \\
& \multicolumn{2}{c}{$I_{\mathrm{C}}$} & \multicolumn{2}{c}{$\rho$} \\
\cline { 2 - 7 } & Estimate & Pvalue & Estimate & Pvalue & Estimate & Pvalue \\
\hline$s_{c}$ & $\mathbf{9 . 4 1 e - 0 2}$ & $<0.001$ & $-6.59 \mathrm{e}-02$ & 0.26 & $\mathbf{0 . 1 4}$ & $<0.001$ \\
$N_{\text {morph }}$ & $\mathbf{- 1 . 0 3 e - 0 3}$ & $<0.001$ & $\mathbf{- 2 . 6 0 e - 0 3}$ & 0.04 & $\mathbf{2 . 5 2 e - 0 3}$ & $<0.001$ \\
\hline$s_{C}$ & $\mathbf{8 . 4 0 e - 0 2}$ & $<0.001$ & $6.17 \mathrm{e}-02$ & 0.34 & $\mathbf{0 . 1 6}$ & $<0.001$ \\
$N_{\text {morph }}$ & $\mathbf{- 1 . 2 4 e - 0 3}$ & $<0.001$ & $\mathbf{- 1 . 3 9 \mathrm { e } - 0 3}$ & 0.284 & $\mathbf{2 . 6 7 e - 0 3}$ & $<0.001$ \\
Number of patches & $\mathbf{1 . 4 8 e - 0 2}$ & $<0.001$ & $\mathbf{- 7 . 3 0 e - 0 2}$ & $<0.001$ & $-8.88 \mathrm{e}-03$ & 0.10 \\
\hline
\end{tabular}

Note: Multivariate linear models (least-square estimation) : Term $\sim s_{c}+N_{\text {morph }}$, and Term $\sim s_{c}+N_{\text {morph }}+$ Number of patches. Estimates that are significantly different than 0 are in bold (Pvalue of the t-test $<0.05$ ). 


\section{FIGURES}

A Without colour shift
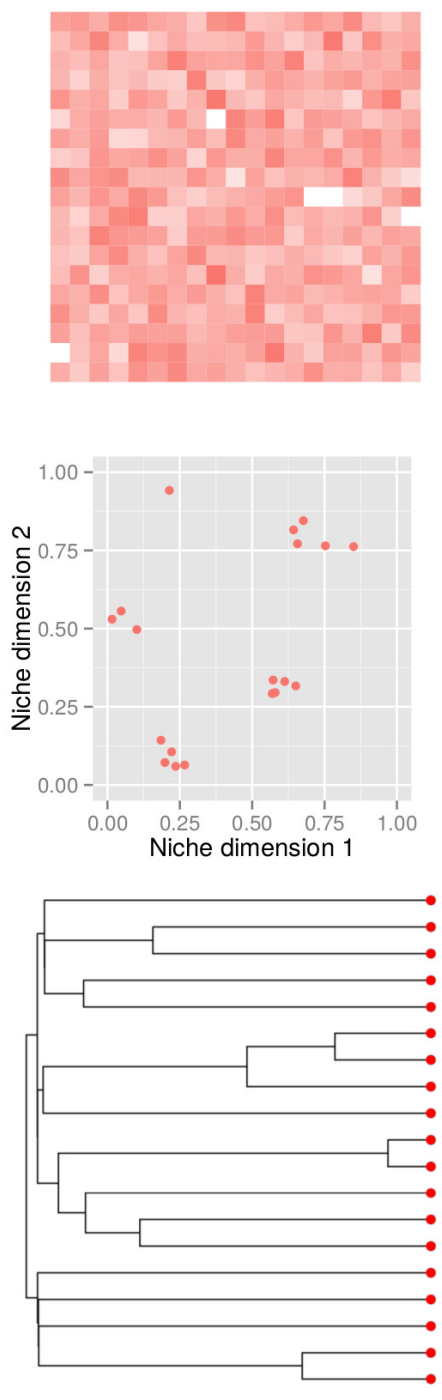

B With colour shift
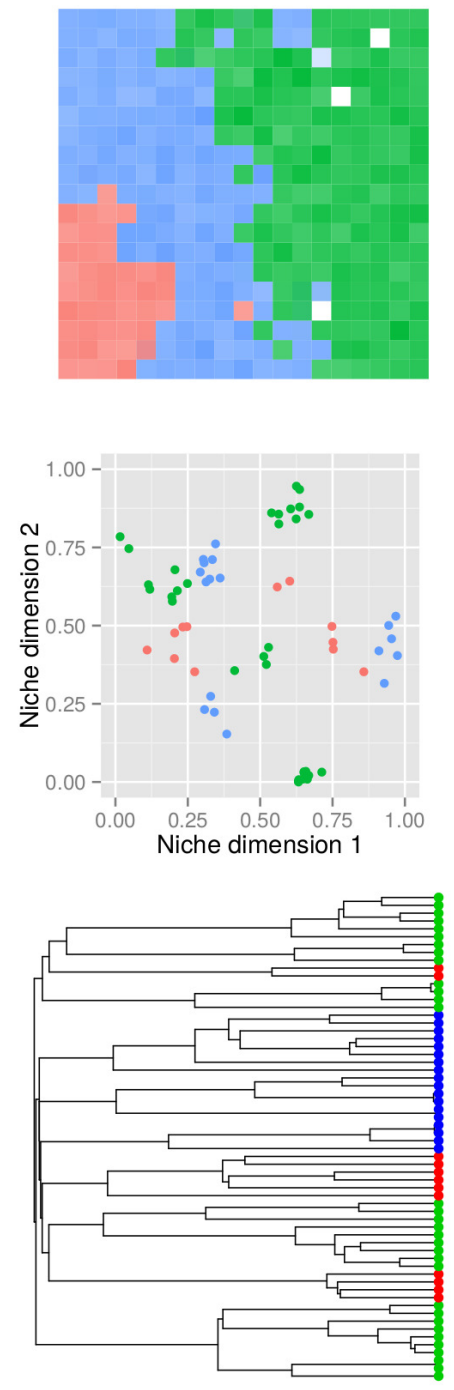

Figure 1: Output of the model after 5 million generations. Example of two simulations assuming either (A) no speciation associated with colour pattern shift $\left(s_{c}=0\right)$, or $(\mathrm{B})$ a proportion of $50 \%$ of speciation events associated with colour pattern shift $\left(s_{c}=0.5, N_{\text {morph }}=30\right)$. From top to bottom, we plot the distribution of the morphs in the grid (the opacity is proportional to the population density in the cell), the occupation of the niche space by the species (represented by dots), and the resulting phylogenies. Each morph is plotted as a distinct colour. In (B), the resulting mosaic is composed of three patches - defined as contiguous cells $(>5$ cells) where individuals share the same morph. Parameter values: see Table 1. 
A

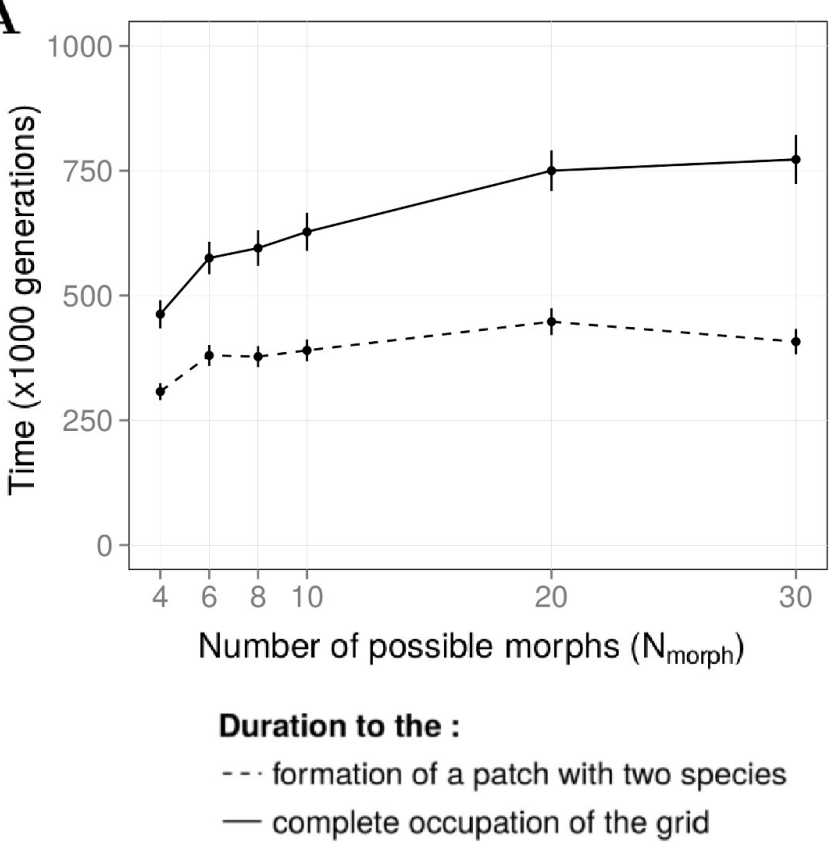

B

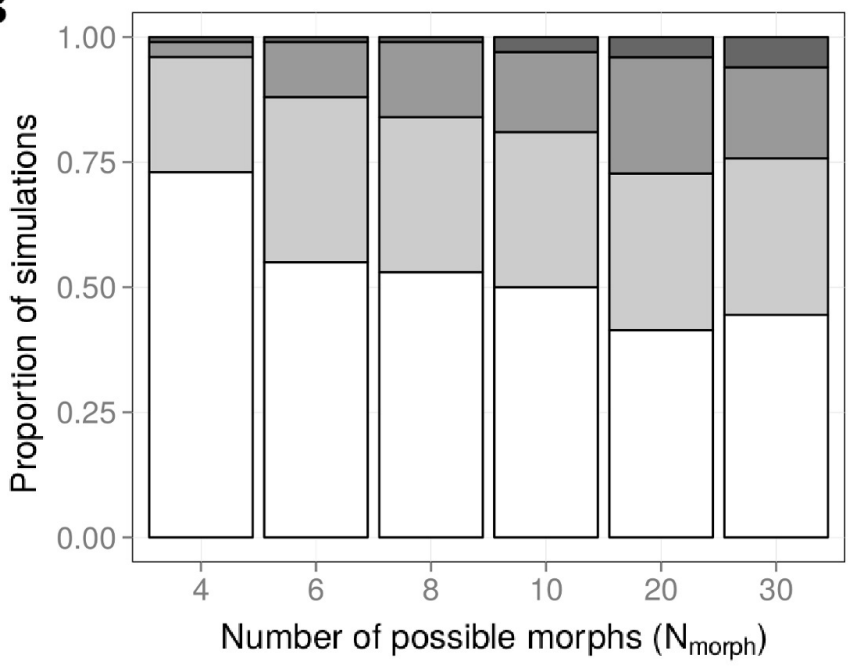

Number of patches :

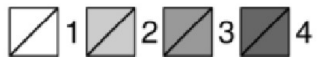

Figure 2: Effects of the number of possible morphs $\left(N_{\text {morph }}\right)$ on the formation of the spatial mosaic of morphs, when there is predation, and when there are colour shifts $\left(s_{c}=0.5\right)$. We plot the numbers of generations until the formation of the first patch with two species and until the complete occupation of the grid (represented by mean values and their confidence intervals) (A), and the proportion of simulations resulting in mosaics with different numbers of patches (represented by different shades of grey) after 5 million generations (B). Parameter values: see Table 1. 


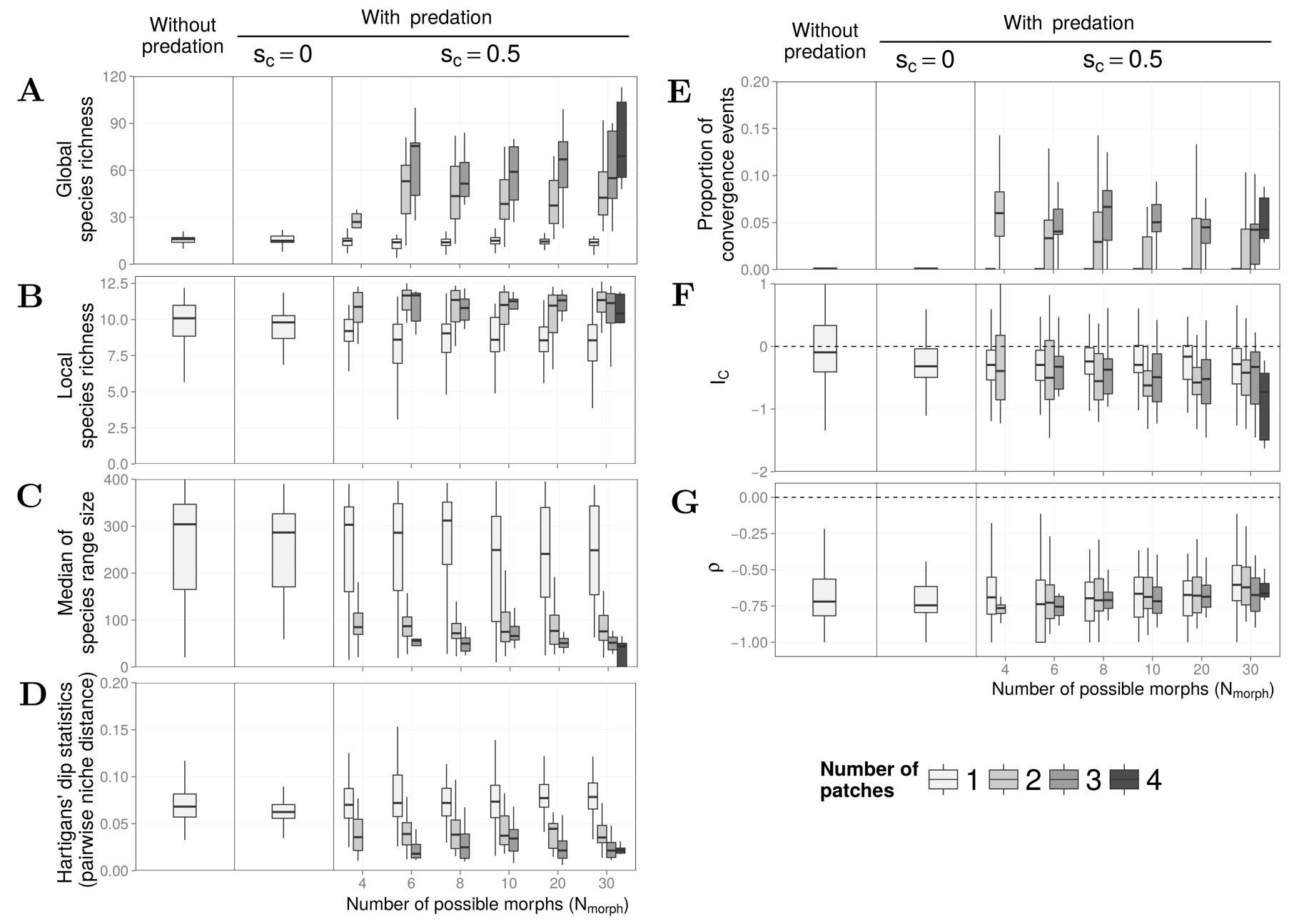

Figure 3: Relationship between the number of patches of the mosaic emerging from the simulations and the spatial and ecological structure of the prey community after 5 million generations. Effects of the proportion of speciation events with colour shift $\left(s_{c}\right)$ and of the number of possible morphs $\left(N_{\text {morph }}\right)$. In each subfigure, from left to right, the pannels corresponds to simulations without predation $\left(p_{p}=0\right)$, to simulations with predation and without colour shift $\left(p_{p}=0.9, s_{c}=0\right)$, and to simulations with predation and with colour shift $\left(p_{p}=0.9\right.$, $\left.s_{c}=0.5\right)$. We plot the number of species of the simulated clades - global (A) and local (B) species richnesses -, the median of species range size (number of cells occupied) (C), the species' niche occupation (Hartigans' dip statistic of the distribution of pairwise niche distance) (D), the proportion of convergence events (E), the estimator of the imbalance $I_{\mathrm{C}}$ of the resulting trees using normalized Colless' index $(\mathrm{F})$, and the temporal shift in diversification rate $\rho(\mathrm{G})$. The dotted lines in the graphs representing the values of $I_{\mathrm{C}}$ and $\rho$ correspond to the expected values under the Yule model (pure birth model). For each combination of parameters, simulations are classified according to the number of patches of their resulting mosaics (different shades of grey). The number of patches has a large 'effect' on the macro-evolutionary pattern. Yet, note that simulations leading to patchy mosaics are relatively rare (see Fig. 2). Data distribution is represented by box-and-whiskers plot. The central line, the box upper/lower limits and the line upper/lower limits correspond to the median, the upper/lower quartile, and the maximum/minimum, respectively. Parameter values: see Table 1. 


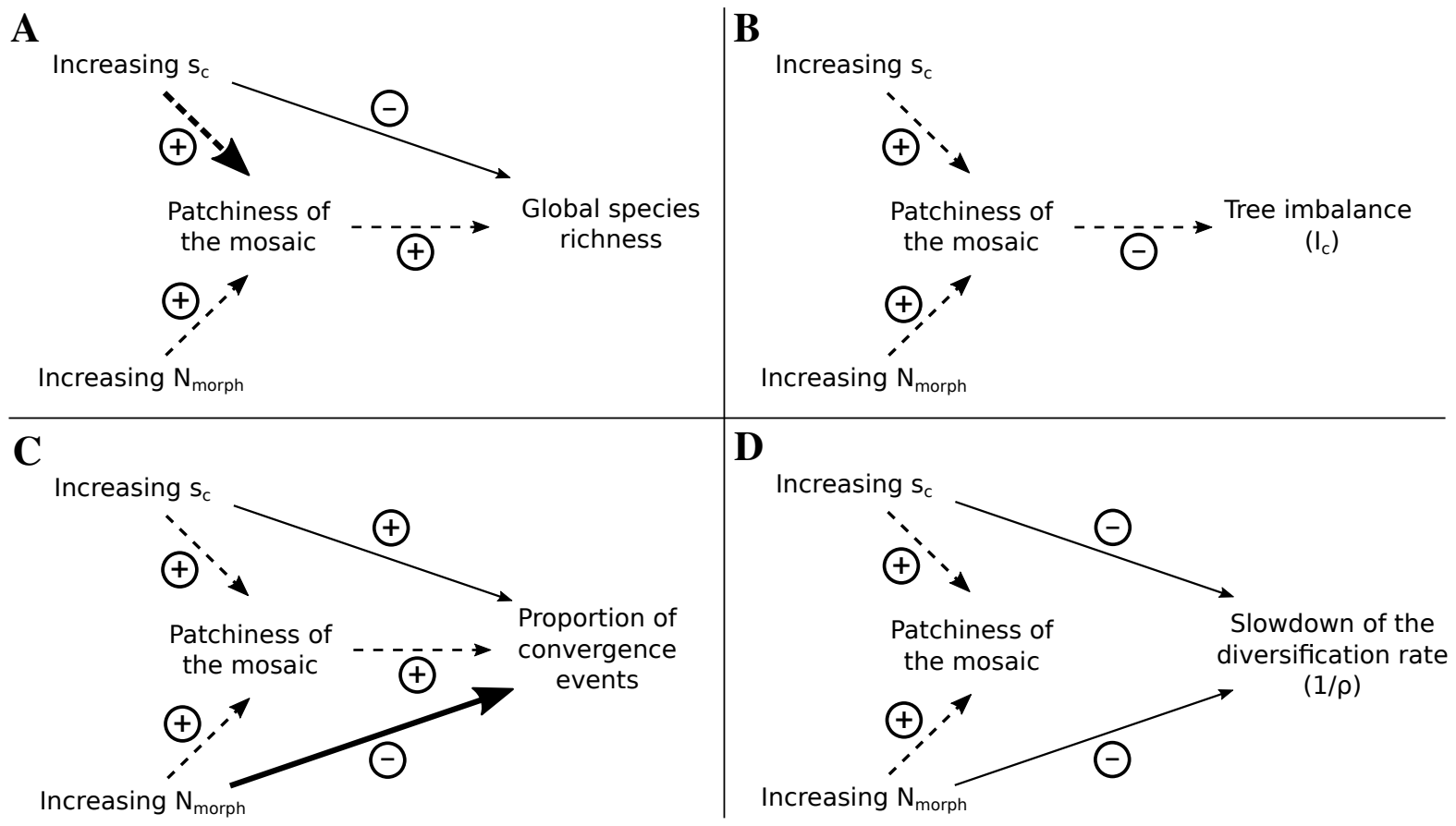

Figure 4: Direct (plain lines) and indirect (via the number of patches of the mosaic, dotted lines) effects of $s_{c}$ and $N_{\text {morph }}$ on the global species richness $(\mathrm{A})$, tree imbalance $\left(I_{\mathrm{C}}\right)(\mathrm{B})$, the proportion of convergence events observed (C), and the slowdown in diversification rate $(1 / \rho)(\mathrm{D})$. Positive and negative forces are represented by $(+)$ and $(-)$ symbols, respectively. When the direct and indirect forces are opposite, the dominant force is represented by a thicker arrow. 


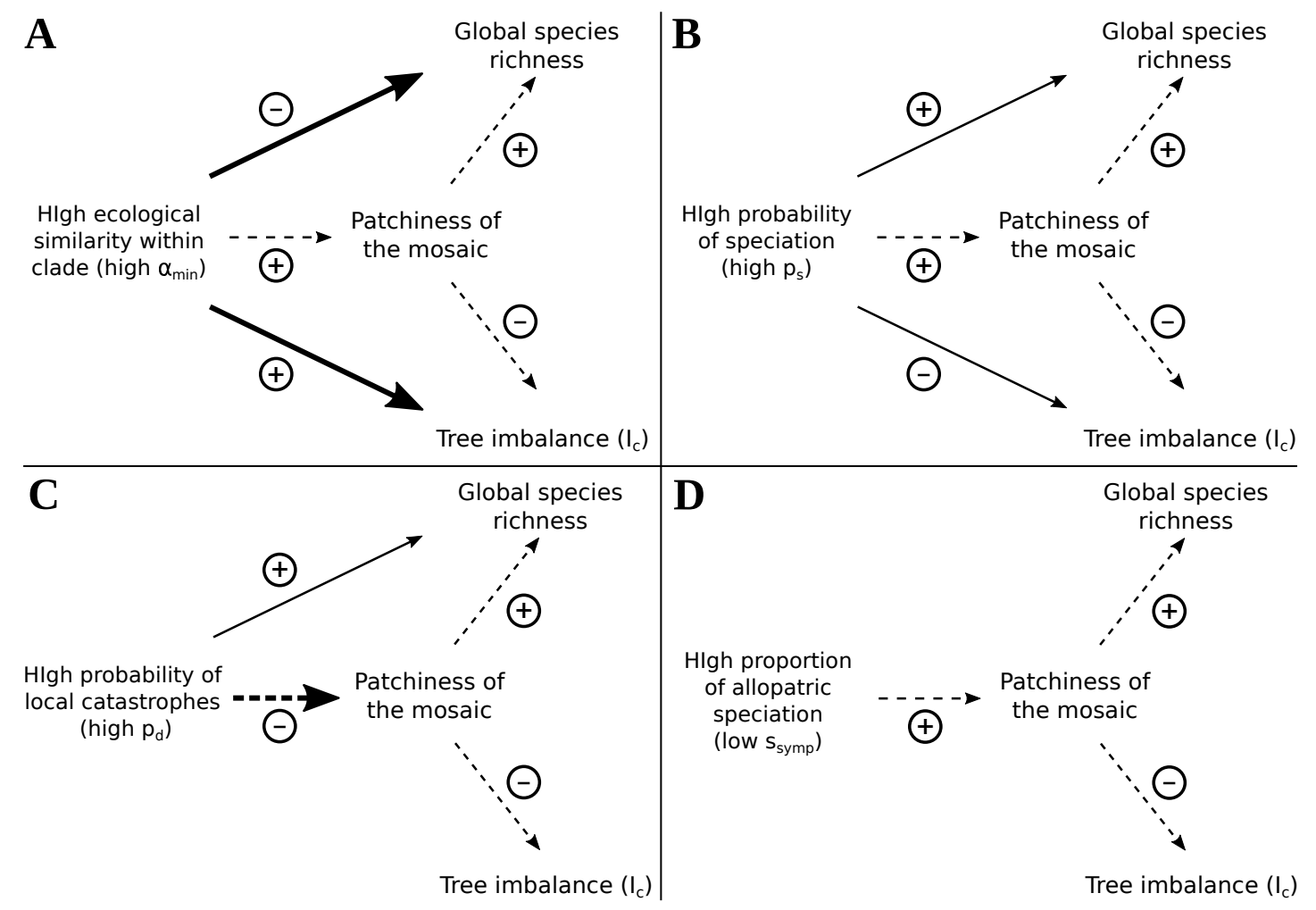

Figure 5: Direct (plain lines) and indirect (via the number of patches of the mosaic, dotted lines) effects of other parameters on the global species richness and on tree imbalance (see supplementary figures S13, S11, S10 and S7 for more details). Positive and negative forces are represented by $(+)$ and $(-)$ symbols, respectively. When the direct and indirect forces are opposite, the dominant force is represented by a thicker arrow. 